\title{
Stabilization and functionalization of iron oxide nanoparticles for biomedical applications $\dagger$
}

\author{
Esther Amstad, ${ }^{a}$ Marcus Textor ${ }^{a}$ and Erik Reimhult ${ }^{* a b}$ \\ Received 14th February 2011, Accepted 11th April 2011 \\ DOI: $10.1039 / \mathrm{c} 1 \mathrm{nr} 10173 \mathrm{k}$
}

\begin{abstract}
Superparamagnetic iron oxide nanoparticles (NPs) are used in a rapidly expanding number of research and practical applications in the biomedical field, including magnetic cell labeling separation and tracking, for therapeutic purposes in hyperthermia and drug delivery, and for diagnostic purposes, e.g., as contrast agents for magnetic resonance imaging. These applications require good NP stability at physiological conditions, close control over NP size and controlled surface presentation of functionalities. This review is focused on different aspects of the stability of superparamagnetic iron oxide NPs, from its practical definition to its implementation by molecular design of the dispersant shell around the iron oxide core and further on to its influence on the magnetic properties of the superparamagnetic iron oxide NPs. Special attention is given to the selection of molecular anchors for the dispersant shell, because of their importance to ensure colloidal and functional stability of sterically stabilized superparamagnetic iron oxide NPs. We further detail how dispersants have been optimized to gain close control over iron oxide NP stability, size and functionalities by independently considering the influences of anchors and the attached sterically repulsive polymer brushes. A critical evaluation of different strategies to stabilize and functionalize core-shell superparamagnetic iron oxide NPs as well as a brief introduction to characterization methods to compare those strategies is given.
\end{abstract}

${ }^{a}$ Laboratory for Surface Science and Technology, ETH Zurich, Wolfgang-Pauli-Strasse 10, CH-8093 Zurich, Switzerland. E-mail: erik. reimhult@boku.ac.at

${ }^{b}$ Department of Nanobiotechnology, University of Natural Resources and Life Sciences (BOKU), Muthgasse 11/II, A-1190 Vienna, Austria $\uparrow$ This article was submitted as part of a collection of articles on surface nanotechnology for biological applications. Other papers on this topic can be found in issue 2 of vol. 3 (2011). This issue can be found from the Nanoscale homepage [http://www.rsc.org/nanoscale].

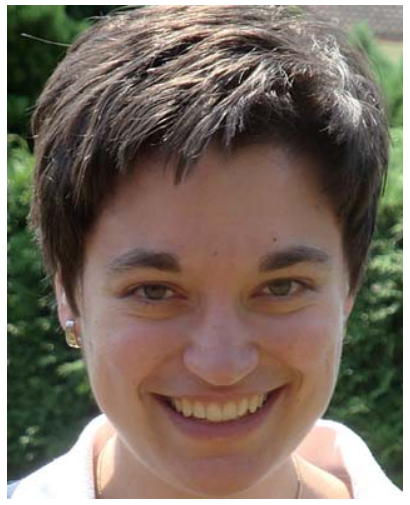

Esther Amstad
Esther Amstad received her $\mathrm{MSc}$ in material science from ETH Zurich (Switzerland) in 2007. She performed her PhD entitled "Surface Functionalizing Superparamagnetic Iron Oxide Nanoparticles Using Nitrocatechol Anchors" under the supervision of Prof. Dr Erik Reimhult and Prof. Dr Marcus Textor at ETH Zurich (Switzerland) where she graduated in 2010. Since April 2011, she has been a Postdoctoral fellow in the experimental soft condensed matter group of Prof. Dr David

A. Weitz at Harvard University. Her current research interests include the assembly and characterization of nanoparticles and microcapsules used as drug delivery vehicles.

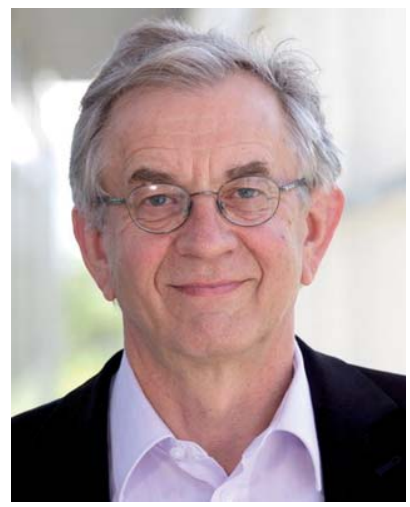

Marcus Textor
Trained in chemistry at the University of Zurich, Marcus Textor spent two years as a Postdoctoral fellow at the School of Molecular Sciences, University of Sussex, UK, working in the field of catalytic model reactions at single-crystal surfaces. In 1978 he took up a position at Alusuisse $R \& D$ Laboratories, Switzerland, finally with world-wide research responsibilities for the fabrication and application of aluminium and composites in the industrial, automotive and packaging sectors. In 1994 he joined ETH Zurich, Department of Materials, establishing an interdisciplinary research group and teaching activities in the area of surfaces and interfaces of light metals and biomaterials. 


\section{Introduction}

Superparamagnetic iron oxide NPs, with core diameters between 3 and $15 \mathrm{~nm}$, are used in a rapidly expanding number of research and practical applications in the biomedical field; the most common include magnetic cell labeling, ${ }^{1,2}$ separation $^{3}$ and tracking, ${ }^{3}$ for therapeutic purposes in hyperthermia ${ }^{4,5}$ and drug delivery, ${ }^{6}$ and for diagnostic purposes, e.g., as contrast agents for magnetic resonance imaging $(\mathrm{MRI})^{7-9}$ (Fig. 1). Superparamagnetic iron oxide NPs are considered to be benign to the body ${ }^{7,10}$ mainly because iron oxide is dissolved under acidic conditions. The resulting $\mathrm{Fe}^{3+}$ ions can be fed into the natural iron storage which is $3-5 \mathrm{~g}$ iron for an adult human. ${ }^{11,12}$ Thus the additional amount of iron released from dissolved iron oxide NPs is negligible if iron oxide NP concentrations in the $\mu \mathrm{g} \mathrm{kg}^{-1}$ body weight range are injected. ${ }^{13}$ Most other magnetic materials such as Co have a higher saturation magnetization $\left(M_{\mathrm{s}}\right)$ compared to iron oxide ${ }^{14}$ and would therefore show a stronger magnetic response, but are toxic, which provides iron oxide with a decisive advantage for the majority of biomedical applications. ${ }^{15}$

To enable that superparamagnetic iron oxide NPs can be dispersed in aqueous media and at physiologic salt concentrations, iron oxide cores are coated with polymers, so-called dispersants. Without a polymer shell NPs will rapidly aggregate through interactions between themselves or with biological molecules and precipitate out of solution. Commercially available superparamagnetic iron oxide NPs intended for magnetic labeling, cell separation purposes and as MR contrast agents are typically coated with sugars such as dextran or synthetic polymers such as silicone. ${ }^{16}$ The reversible adsorption of these dispersants which have molecular weights $\left(M_{\mathrm{w}} \mathrm{s}\right) \geq 10 \mathrm{kDa}$ is due to poor affinity of the repeat units towards iron oxide. ${ }^{17}$ Therefore these dispersants often enwrap and cluster multiple iron oxide NP cores by direct physisorption to multiple NP surfaces. However, the control over cluster size is poor (Fig. 2a). ${ }^{18-21}$

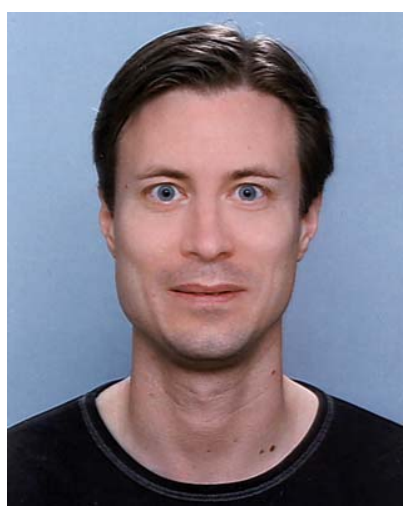

Erik Reimhult
Erik Reimhult received his $P h D$ in Physics on the self-assembly of supported lipid bilayers and combinatorial sensor design in 2004 under the supervision of Profs Bengt Kasemo and Fredrik Höok. Two postdoctoral stays on nanofabrication, colloidal and amphiphile assembly at the Institute of Materials Research and Engineering in Singapore with Prof. Wolfgang Knoll and ETH Zurich with Prof. Marcus Textor were followed by a junior group leader position with Prof. Marcus Textor in 2008 and appointment to full professor at the University of Natural Resources and Life Sciences Vienna in Nanobiotechnology of Supramolecular materials in 2010.
Alternatively, superparamagnetic iron oxide NPs can be stabilized with low $M_{\mathrm{w}}<10 \mathrm{kDa}$ dispersants consisting of a polymer spacer with a covalently bound anchor that has high affinity for the NP surface (Fig. 2b). Dispersants self-assemble on the NP surface in a well-defined adsorption geometry through anchors. The resulting core-shell NPs can be divided into four components, namely the core, anchor, spacer and optional surface functionalities (Fig. 2b). Each of these components can independently be adjusted through the modular build-up and defined geometry, rendering such NPs very versatile for a multitude of applications..$^{22,23}$ One of the resulting advantages of this modular and controlled build-up is that the hydrodynamic size of these NPs can be precisely controlled in contrast to NPs with dispersant shells consisting of physisorbed high $M_{\mathrm{w}}$ dispersants. Furthermore, the well-defined assembly of dispersants at the NP surface enables controlled surface presentation of functionalities. NP size, stability, dispersant shell thickness and control over functionalities presented at the NP surface are the factors that critically determine NP performance in the demanding environment of a biological fluid. ${ }^{24,25}$ These parameters are largely determined by the dispersant shell of NPs. Therefore, dispersants are of pivotal importance for the performance and further development of NPs.

This review is focused on different aspects of NP stability, from its practical definition to its implementation by molecular design of the surrounding shell and further on to its influence on the magnetic properties of the superparamagnetic iron oxide NPs. In order to define and investigate NP stability a comprehensive set of complementary characterization techniques are required which will first be described and compared. Thereafter, different de facto definitions of NP stability in the literature will be discussed. After these general aspects, the review describes the surface modification of superparamagnetic iron oxide NPs with dispersants of low and high surface affinity and $M_{\mathrm{w}}$. Special attention is given to the selection of anchors because of their importance for the surface modification and stability of sterically stabilized NPs. In particular, we focus on catechol derived anchors as they combine high binding affinity to $\mathrm{Fe}_{3} \mathrm{O}_{4}$ surfaces and low desorption rates if properly modified. ${ }^{23}$ This review further details how dispersants have been optimized to gain close control over iron oxide NP stability, size and functionalities by independently considering the influences of anchors and spacers, and compares different strategies to functionalize core-shell NPs. The review ends with insights into the influence of the stability of superparamagnetic iron oxide NPs, and therefore the strategy for iron oxide NP stabilization and functionalization, on their magnetic properties.

\section{Characterization of nanoparticles}

To closely control the assembly of dispersants on the NP surface and understand its influence on NP size distribution, stability and functionality, thorough characterization is essential. In addition, to understand the relation between core size, selfassembly of dispersants at the NP surface, shell thickness and NP stability, further parameters such as the dispersant packing density, core size distribution and core surface roughness have to be considered. 


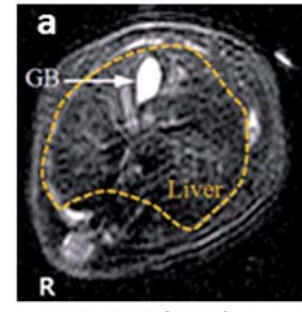

pre-contrast

\section{c}

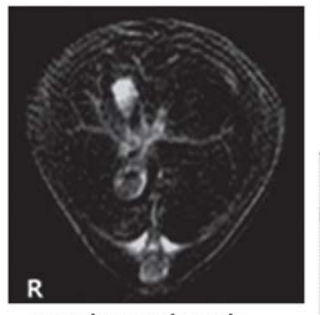

post-contrast
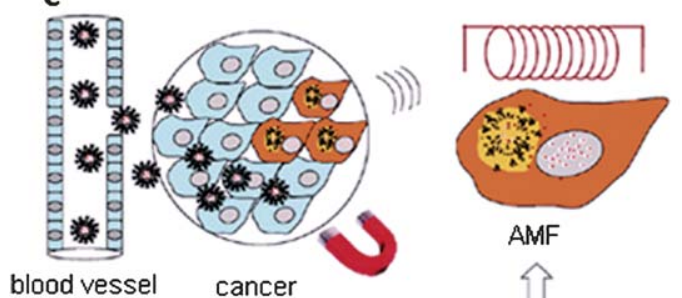

AMF

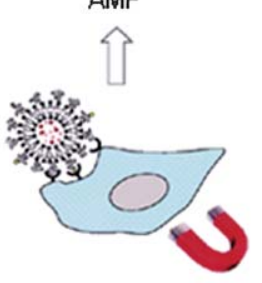

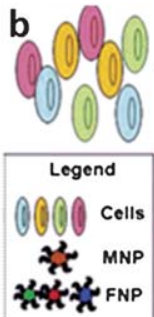
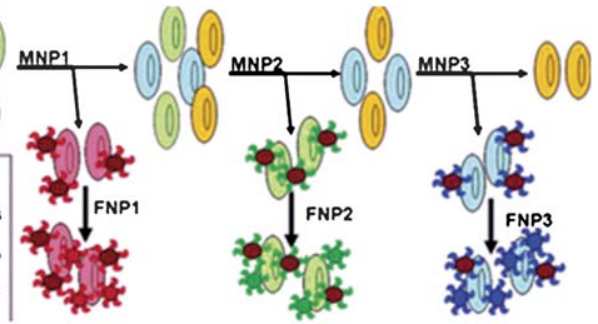

d
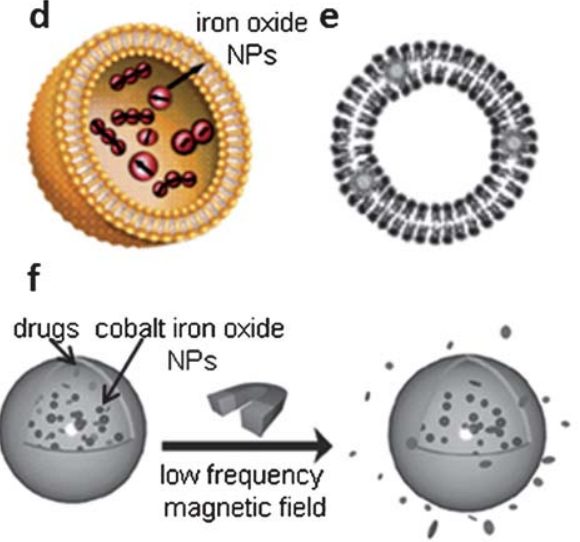

Fig. 1 Biomedical applications of superparamagnetic iron oxide NPs. Superparamagnetic iron oxide NPs can be used for diagnostic purposes as (a) magnetic resonance (MR) contrast agent ${ }^{200}$ (Magnetic resonance in medicine by International Society for Magnetic Resonance in Medicine. Reproduced with permission of JOHN WILEY \& SONS, INC. in the format Journal via Copyright Clearance Center.), (b) for cell separation purposes either as magnetic NPs (MNPs) or as fluorescently labeled NPs (FNPs) and for therapeutic reasons ${ }^{201}$ (Analytical chemistry by American Chemical Society. Reproduced with permission of AMERICAN CHEMICAL SOCIETY in the format Journal via Copyright Clearance Center.), (c) in hyperthermia ${ }^{202}$ (Journal of controlled release by Controlled Release Society. Reproduced with permission of ELSEVIER BV in the format Journal via Copyright Clearance Center.) and in drug delivery vehicles where they (d) can be incorporated into the lumen of liposomes ${ }^{203}$ (reprinted by permission of IOP Publishing Ltd) or into the bilayer of liposomes. ${ }^{204}$ (ACS nano by American Chemical Society. Reproduced with permission of AMERICAN CHEMICAL SOCIETY in the format Journal via Copyright Clearance Center.) Superparamagnetic iron oxide NPs were shown to trigger release if a (e) high and (f) low frequency magnetic field is applied. ${ }^{205}$ (Reproduced by permission of The Royal Society of Chemistry.)

The core size, size distribution and morphology can be characterized with a combination of different techniques such as transmission electron microscopy (TEM), ${ }^{26} \mathrm{X}$-ray diffraction $(\mathrm{XRD})^{26}$ and scattering techniques such as small angle $\mathrm{X}$-ray scattering $(\mathrm{SAXS})^{27}$ and small angle neutron scattering (SANS). ${ }^{\mathbf{2 8 , 2 9}}$ The hydrodynamic diameter of dispersed NPs (the effective diameter of the NP when diffusing in water, typically understood as the sum of the core diameter and twice the shell thickness) can be assessed with scattering techniques, e.g., dynamic and static light scattering (DLS and SLS), ${ }^{30}$ SANS $^{29}$ and X-ray disc centrifugation (XDC). ${ }^{31}$ The dispersant packing density can be quantified with thermogravimetry analysis (TGA) ${ }^{23,32}$ and SANS. ${ }^{29}$ To verify that dispersants rather than impurities or capping agents such as oleic acid are adsorbed on the NP surface, and thus to assign the mass loss measured with TGA to the dispersants adsorbed on the NP surface, stabilized NPs can be analyzed e.g. with Fourier transform infrared (FTIR) spectroscopy ${ }^{32,33}$ or, less commonly used, X-ray photoelectron spectroscopy (XPS). ${ }^{23}$ To extract information about the dispersant packing density and dispersant density profile from SANS
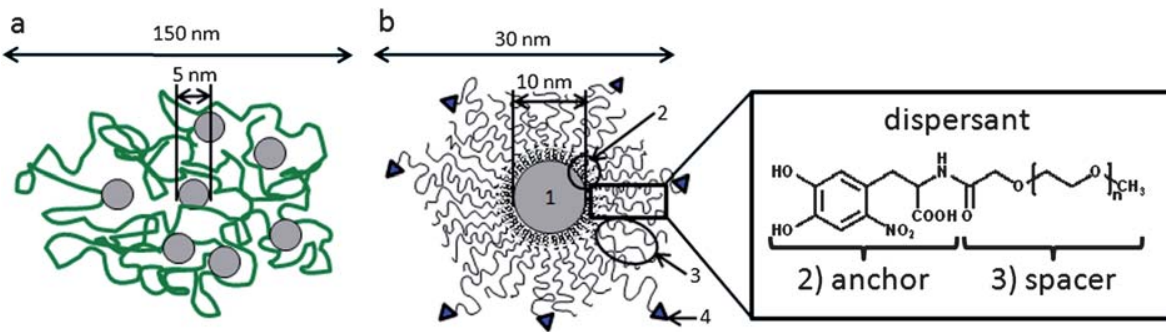

Fig. 2 Steric stabilization of iron oxide NPs. (a) Schematic of commercially available iron oxide based MR contrast agents such as Feridex and Endorem. Superparamagnetic iron oxide NPs are coated with physisorbed dispersants such as dextran. The poor binding affinity of dextran to iron oxide leads to reversible adsorption of dextran on the iron oxide NP surface. Furthermore, multiple iron oxide cores are embedded in one cluster. The resulting hydrodynamic diameter is many times larger than the core diameter. (b) Superparamagnetic iron oxide NPs stabilized with low $M_{\mathrm{w}}$ dispersants result in core-shell iron oxide NPs. These NPs can be divided into four components namely (1) core, (2) anchors, (3) spacers and optionally (4) functionalities. 
results, it is highly beneficial to do contrast variation experiments, where the contrast of the core and shell is varied by changing the ratio of protonated to deuterated solvents and thus varying the scattering length density of the solvent. ${ }^{34}$ Alternatively, information about the dispersant packing density on NP surfaces can be extracted from SANS results acquired with polarized neutrons. ${ }^{29}$ Furthermore, the dispersant density profile can be assessed with SANS measurements. ${ }^{35}$

Because of the different advantages and disadvantages of each characterization technique, it is highly beneficial to characterize NPs with multiple, complementary methods. However, if results obtained with different methods are directly compared, attention has to be paid to the precise meaning of the results. Differences and artifacts can be introduced for example through different weighting of sizes, model-dependent extraction of parameters and through sample preparation protocols. Such aspects can lead to substantial differences in the quantification of a physical property of the NPs with different techniques.

Scattering techniques reveal intensity weighted averages $\left(I(q) \propto r^{6}\right)$ and are thus sensitive to large NPs and clusters. X-ray diffraction (XRD) reveals volume weighted averages $\left(\propto r^{3}\right)$ while TEM allows direct visualization of number weighted $\left(\propto r^{1}\right)$ structures. To compare scattering to TEM results for the mean size, the weighting of the sizes has therefore to be taken into account. This is only possible, if the core size distribution is known.

TEM reveals direct, model independent information. Preparation of NP samples for TEM is done through drying on, e.g., carbon supported TEM grids, unless TEM samples are prepared with cryo-preparation techniques. Drying of NP dispersions can introduce artifacts such as NP agglomeration and inhomogeneous assembly of NPs of different sizes. Furthermore, it leads to a collapse of the dispersant layer that makes it impossible to accurately determine the wet shell thickness even if the coating can be visualized with TEM. ${ }^{36}$

In contrast, scattering techniques allow analyzing NPs directly in dispersion and are therefore less prone to sample preparation artifacts and better suited to determine the shell thicknesses. However, they require model dependent data analysis. SANS and SAXS data are fitted with form factors. The form factors assume a certain structure and size distribution of the evaluated objects. Therefore, accurately done, the data analysis requires prior knowledge about the shape and structure of the analyzed objects. By comparing scattering data to a set of models the dispersant shell density profile can be obtained if the quality of the scattering data is sufficient. However, the NP concentration can critically affect the outcome of scattering results. If the NP concentration is high, multiple scattering significantly influences light scattering results. ${ }^{37}$ SANS and SAXS data acquired on highly concentrated NP dispersions typically comprise a structure factor contribution that is convoluted with the form factor. ${ }^{38}$ While the form factor describes the size distribution and shape of $\mathrm{NPs}$, the structure factor is influenced by inter-particle interactions, clustering and assembly of NPs. Because multiple scattering and structure factor contributions can significantly influence scattering results, it is very important to prepare samples such that effects of the NP concentration on the scattering results can be excluded or appropriately accounted for.

The fact that the analysis of data acquired with scattering techniques is model dependent renders a comparison to data acquired with complementary, model independent techniques highly advantageous. The comparison of the quantification of NP parameters obtained with scattering techniques with quantification measured with model-independent techniques for the same parameter allows checking the validity of the model assumed to analyze scattering data.

The value of characterizing NPs with different, complementary techniques can be exemplified on the packing density and density profile of dispersants adsorbed on NP surfaces. These parameters can be measured and quantified with SANS. However, to analyze SANS data, a form factor that comprises assumptions about the core-shell structure of the NPs including the dispersant density profile has to be applied to analyze the scattering data.

The mass ratio of organic to inorganic materials can be quantified with TGA, although no information on the density profile can be obtained. To ensure that the mass loss of organic molecules measured with TGA can exclusively be assigned to dispersants rather than to impurities or remaining capping agents, further chemical analysis on the stabilized NPs such as FTIR or XPS is required ${ }^{23}$ However, TGA is also sensitive to the morphology of cores as the dispersant packing density is normalized to the NP surface area that is calculated from the mass of NPs assuming a certain core size distribution and shape. Information of the NP size distribution can only partially be obtained from TEM images due to unknown potential segregation effects of NPs during sample preparation. Therefore, the dispersant packing density and density profile on NPs can only be revealed with any certainty if NPs are characterized with multiple methods, such as SANS, TGA, TEM and FTIR spectroscopy.

\section{Nanoparticle stability}

The term "stability" is used with very different meaning in the literature on NPs, mostly without explicit acknowledgement of these differences. NPs are often imprecisely considered stable if they do not visibly precipitate over a finite period of time. ${ }^{39,40} \mathrm{~A}$ thorough characterization of NP dispersions e.g. with scattering techniques allows us to define NP stability more precisely.

The importance of the technique and conditions used to characterize NP stability was exemplified on poly(ethylene glycol)-hydroxydopamine (PEG-hydroxydopamine) stabilized superparamagnetic iron oxide NPs. PEG (0.55 kDa)-hydroxydopamine stabilized iron oxide NPs were stable for more than a year if stored and analyzed at RT. ${ }^{22}$ However, PEG (5 kDa)hydroxydopamine stabilized iron oxide NPs agglomerated if they were subjected to multiple filtrations (Fig. 3). ${ }^{23}$ If dispersants adsorb reversibly at the NP surface, dispersants adsorbed on the NP surface and dispersants free in solution are in equilibrium. If NP dispersions are filtered, free dispersants are removed. To reestablish the equilibrium in the NP dispersion, some of dispersants bound to the NP surface desorb leading to a lower dispersant packing density at the NP surface. The dispersant packing density at the NP surface decreases with increasing times NP dispersions are filtered. If the dispersant packing density at the NP surface drops below a critical value, NPs start to agglomerate. ${ }^{41}$ The fact that PEG (5 kDa)-hydroxydopamine stabilized iron oxide NPs agglomerated if subjected to filtrations 

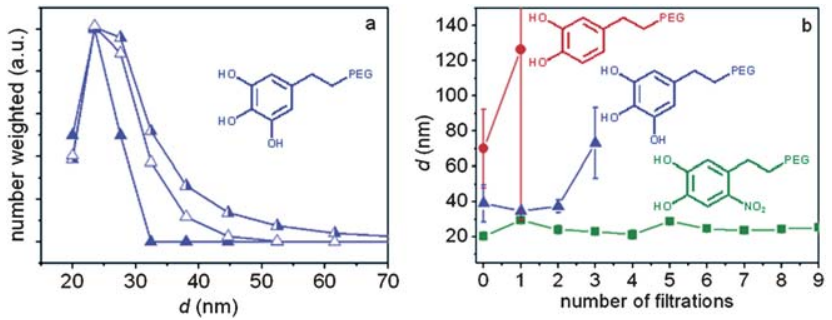

Fig. 3 Characterization of the stability of superparamagnetic iron oxide NPs. The stability of superparamagnetic iron oxide NPs was measured with DLS at $25^{\circ} \mathrm{C}$. (a) The hydrodynamic diameter of PEG $(0.55 \mathrm{kDa})-$ hydroxydopamine stabilized iron oxide NPs as-stabilized (- $\mathbf{\Delta - )}$, after storage for 1 year in PBS (- $\left.\Delta_{-}\right)$and after storing them for 20 months in HEPES (- $\triangle-)^{206}$ (Copyright Wiley-VCH Verlag GmbH \& Co. KGaA. Reproduced with permission.) (b) The stability of superparamagnetic iron oxide NPs was evaluated as a function of the number of filtrations performed to remove excessive dispersants of iron oxide NPs stabilized with PEG (5 kDa)-nitrodopamine (-ם-), PEG (5 kDa)-hydroxydopamine (- $\mathbf{\Delta}-)$ and PEG (5 kDa)-dopamine (- - -). ${ }^{207}$ (Nano letters by American Chemical Society. Reproduced with permission of AMERICAN CHEMICAL SOCIETY in the format Journal via Copyright Clearance Center.) While PEG-hydroxydopamine stabilized iron oxide NPs were stable at RT for more than 20 months, they started to agglomerate after excessive dispersants were removed by more than two filtrations. This indicates that superparamagnetic iron oxide NPs agglomerate upon dilution and will lead to adverse consequences if applied in vivo.

indicates that hydroxydopamine adsorbs reversibly on $\mathrm{Fe}_{3} \mathrm{O}_{4}$ surfaces. However, if the dispersant packing density at the NP surface is sufficiently high under the conditions NP dispersions are stored, then NPs remain long-term stable even if they are stabilized with reversibly binding anchors such as hydroxydopamine (Fig. 3a). These NPs, however, will agglomerate upon dilution (Fig. 3b).

Applications of superparamagnetic iron oxide NPs in vitro in cell cultures and in vivo always include high dilutions of NP dispersions. Therefore, reversible dispersant adsorption that leads to iron oxide NP agglomeration can have severe adverse consequences for these applications. Once injected into a living body, agglomeration of NPs is difficult to assess because NP size cannot be directly measured anymore. Additionally, once NPs are exposed to cells or injected into the body NP agglomeration is convoluted with other effects such as exposure to many different proteins that potentially adsorb on the NP surface or even replace dispersants if dispersants are insufficiently anchored to the NP surface. This then leads to a poorly defined system where analysis of, e.g., the efficiency of targeting NPs to desired locations by the addition of ligands to the NP shell is difficult to separate from effects caused by uncontrolled NP agglomeration and non-specific protein adsorption on the NP surface. This illustrates the necessity to characterize NPs stringently, especially if they are intended for biomedical applications. Additionally, to be encompassing, the criteria for full colloidal stability of NPs should include the NP characterization under dilute conditions and in the presence of sticky macromolecules that are biomedically relevant.

Iron oxide NP stability is typically evaluated based on DLS measurements performed at RT at a certain NP concentration
(Table 1). Especially for in vivo applications, it would be beneficial to measure iron oxide NP stability and size with more thorough characterization methods. One experimentally easily accessible possibility to measure NP stability under demanding conditions is temperature dependent DLS measurements. Such measurements have been shown to provide a good measure of binding affinity and reversibility of dispersants on the iron oxide NP surface, which directly translates into long-term NP stability at highly diluted concentrations under physiological conditions. ${ }^{23}$

In theory, NPs are stable if the inter-particle potential $\left(U_{\text {tot }}\right)$ has an energy barrier that is high compared to $k_{\mathrm{B}} T$. In a first approximation, $U_{\text {tot }}$ of NPs contains four contributions, the attractive van der Waals and magnetic attraction potentials and the repulsive electrostatic and steric potentials. ${ }^{42,43}$ If the core radius, shell thickness, saturation magnetization, $\zeta$ potential and dispersant density profile are known, these potentials can be calculated. ${ }^{42-44}$ Considering the four main contributions to $U_{\text {tot }}$, NPs can be electrostatically or sterically stabilized to prevent agglomeration induced through the attractive van der Waals and magnetic potentials. Optionally, the two stabilization methods can be combined. Electrostatic NP stabilization is only effective at low ionic strength concentrations and at $\mathrm{pHs}$ far above or below the IEP of NPs. However, biomedical applications require NP stability under high salt concentrations and over a range of pHs. Therefore, NPs intended for these applications have to be sterically stabilized. ${ }^{45,46}$ The following section will detail different approaches and advances in steric stabilization of NPs.

\section{Steric stabilization of iron oxide nanoparticles}

Steric stabilization relies on polymers, so-called dispersants, that surround NP cores. Dispersants that yield a sufficiently thick shell around the NP core to overcome the attractive van der Waals and magnetic potentials impart long-term colloidal stability under dilute conditions, high salt concentrations and elevated temperatures. Dispersants used to sterically stabilize superparamagnetic iron oxide NPs can roughly be divided into two groups. One group of dispersants consists of repeat units that have low affinity to the iron oxide NP surface. This leads to reversible dispersant adsorption. The other group of dispersants typically consists of a high affinity anchor that is covalently linked to a low $M_{\mathrm{w}}$ spacer, usually below $10 \mathrm{kDa}$. Optimized dispersants result in good NP stability at high ionic strength, physiological $\mathrm{pH}$ and temperature, which are absolute requirements for biomedical applications.

\subsection{Physisorption of high $M_{\mathrm{w}}$ dispersants}

Superparamagnetic iron oxide NPs used for clinical applications are primarily coated with polymers such as dextran, ${ }^{47}$ alginate ${ }^{48}$ chitosan, ${ }^{49}$ poly(vinyl amine) (PVA) ${ }^{50-52}$ or poly(acrylic acid) (PAA) ${ }^{53}$ or by electrostatically adsorbing charged polymers like poly(ethylene imine) (PEI) to which subsequently a layer of poly (ethylene oxide)- $b$-poly(glutamic acid) (PEO-PGA) can be adsorbed (Fig. 4). ${ }^{54}$ These polymers lack a well-defined high affinity anchor that could irreversibly couple them to the iron oxide NP surface and typically have a molecular weight $>10 \mathrm{kDa}^{9}{ }^{9}$ Therefore, such dispersants often encapsulate 


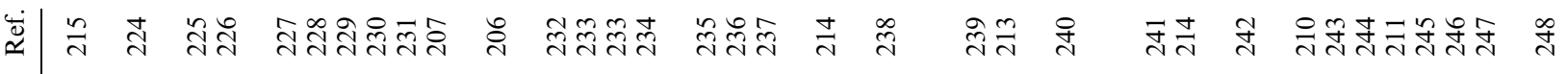
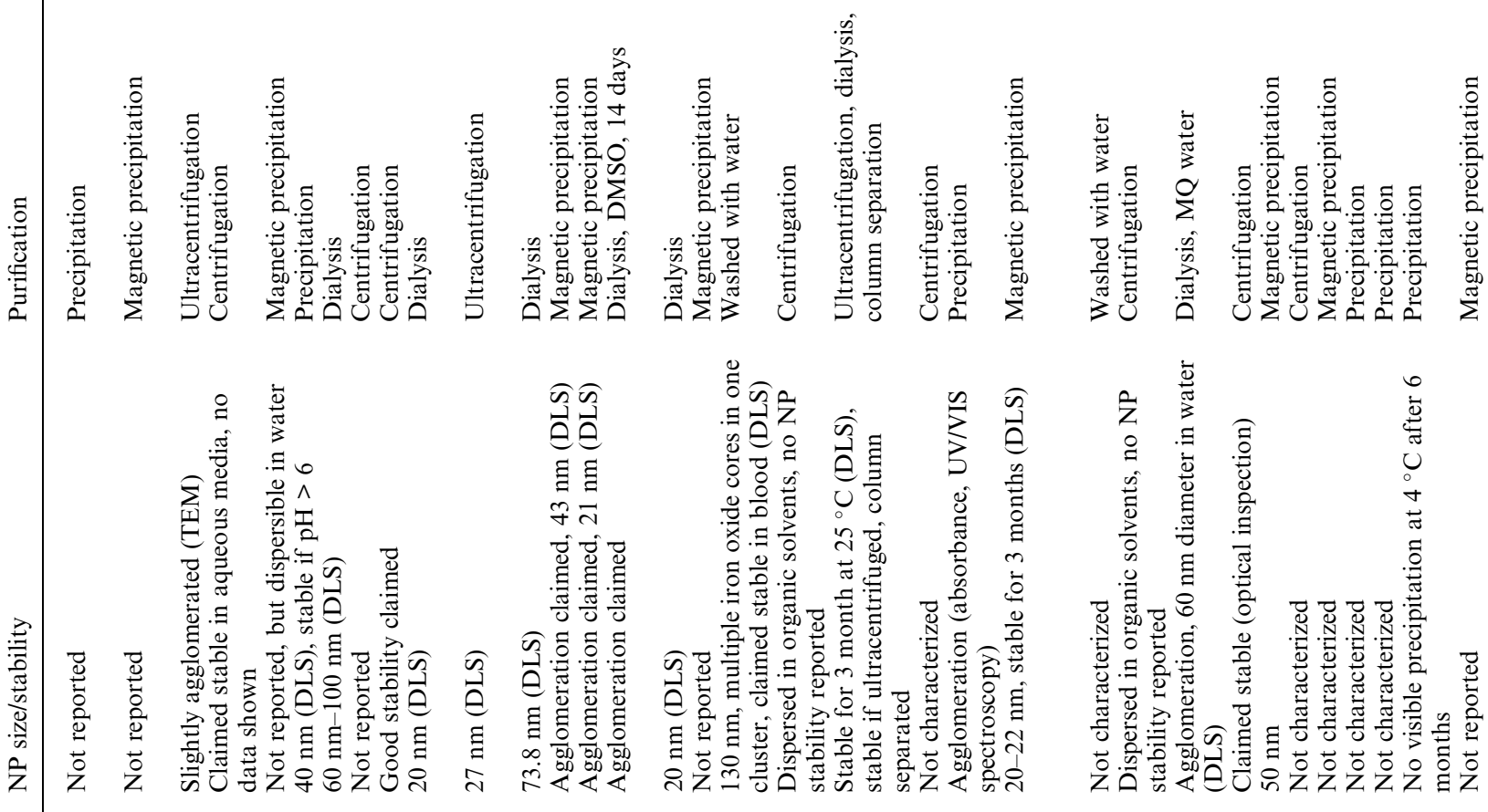

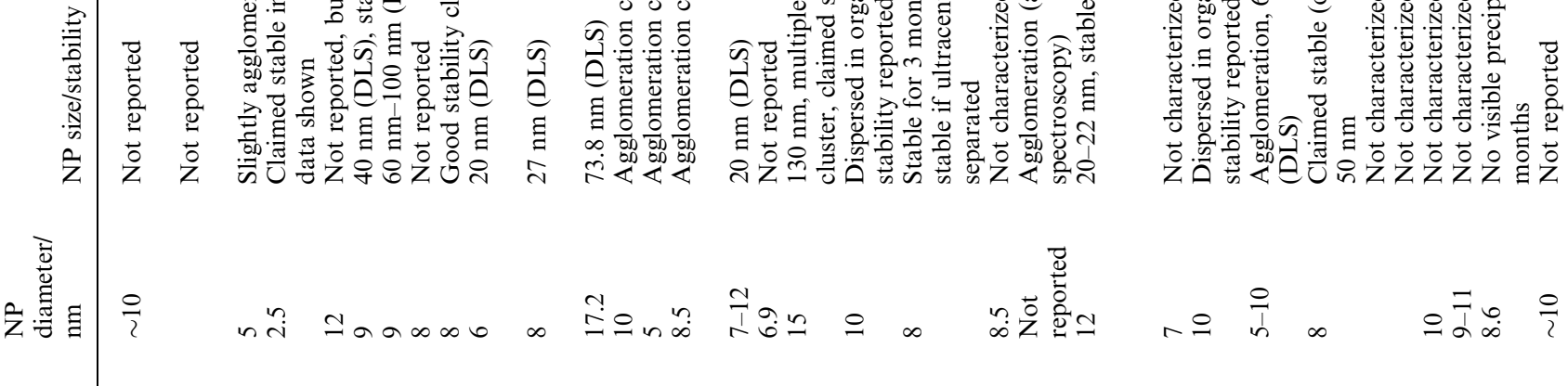

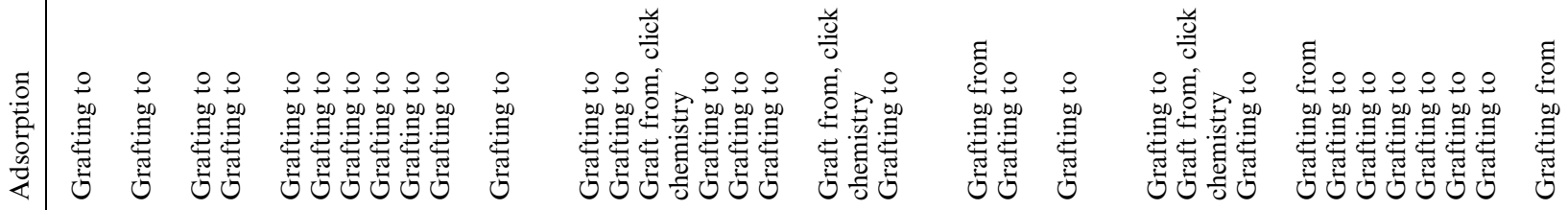

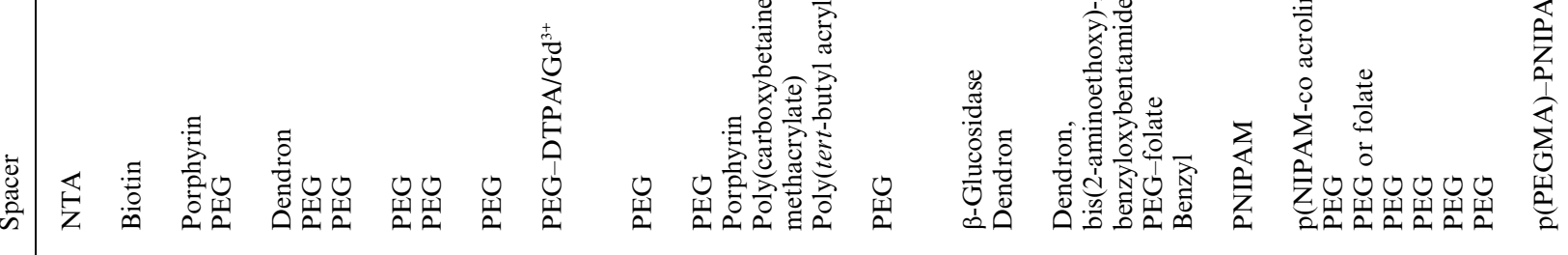

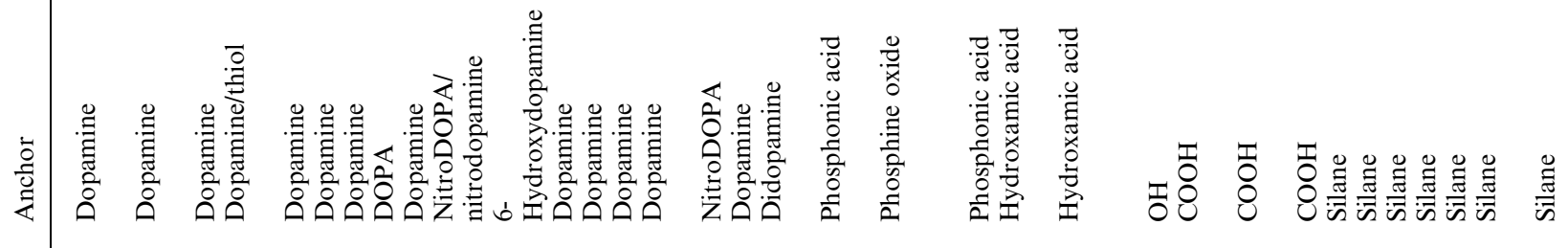
象

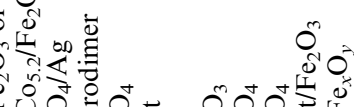

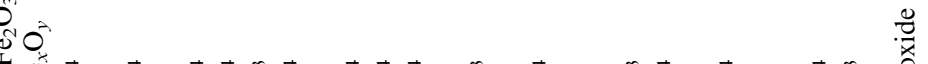




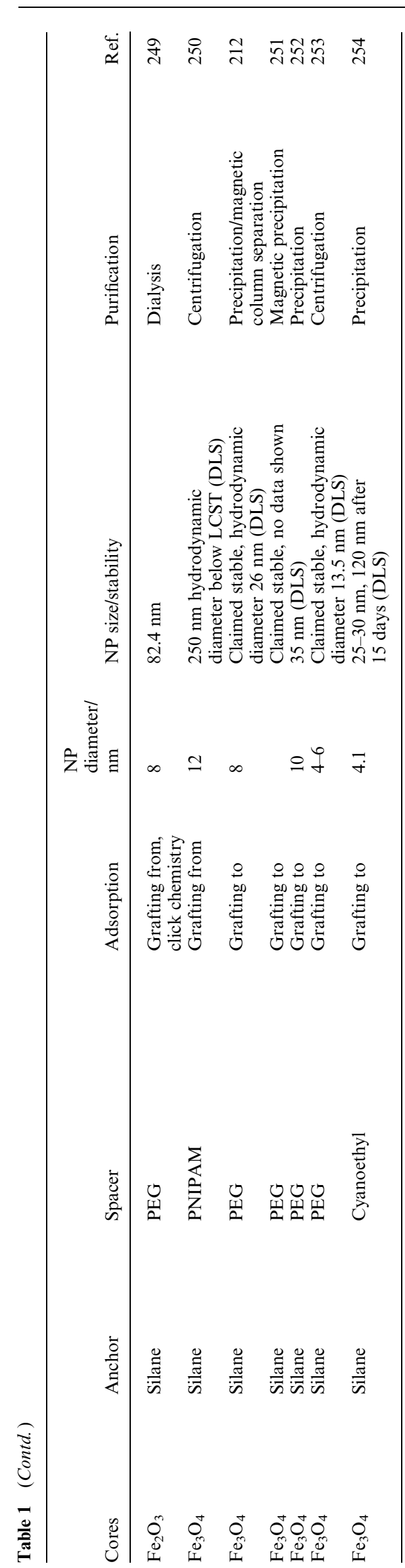

multiple cores within one cluster (Fig. 2a). The resulting hydrodynamic cluster radius is many times larger than the radius of individual cores (Fig. 5). ${ }^{19-21}$ The weak physisorption of the stabilizing polymer dispersants compromises iron oxide NP stability ${ }^{55}$ leads to protein adsorption onto the core particle and drastically decreases blood circulation time if applied in vivo. ${ }^{56}$ The poorly defined NP interface prevents controlled functionalization in terms of number and presentation of ligands. ${ }^{55}$

To prevent desorption of dextran from iron oxide NPs, dextran has been crosslinked after it was adsorbed on the iron oxide NP surface (Fig. 5a). ${ }^{57}$ However, the resulting hydrodynamic diameter was large compared to the core diameter and control over the dispersant layer thickness difficult. Additionally, epichlorohydrin, the crosslinking agent used to immobilize dextran on the iron oxide NPs, is classified as cancerogenic, mutagenic and reproxotic. ${ }^{58,59}$

\subsection{Anchored low $M_{\mathrm{w}}$ dispersants}

As mentioned in the Introduction a desirable alternative in terms of resultant size, interfacial stability and ability to present surface functional groups in a defined manner is low $M_{\mathrm{w}}$ dispersants that consist of one well-defined anchor covalently linked to a spacer (Fig. 2b). A representative set of recent work on superparamagnetic iron oxide NPs stabilized with such dispersants is summarized in Table 1.

Low $M_{\mathrm{w}}$ dispersants can be bound to NP surfaces either through the "grafting to" or the "grafting from" technique (Fig. 6). For the latter approach, initiators are covalently bound to the NP surface. Spacers can subsequently be grown in situ, e.g. by radical polymerization, from initiators that have been attached to NP surfaces (Fig. 6a). ${ }^{60,61}$ This approach results in high dispersant packing densities and therefore good NP stability, because the brush density is set by the density of anchors from which the grafting from is initiated. Although it leads to a desirable high shell density the "grafting from" technique has some inherent drawbacks. Dispersant characterization and control over the dispersant polydispersity and layer thickness are difficult. Furthermore, functionalization of stabilized NPs with different ligands or other functional units and controlling the density of functional groups presented at the NP interface are challenging. ${ }^{62}$

If low $M_{\mathrm{w}}$ dispersants are synthesized prior to their adsorption on the NP surface, they can be characterized with conventional chemical characterization methods. Low $M_{\mathrm{w}}$ dispersants can be grafted to the NP surface without the requirement for in situ chemistry using suitable anchors (Fig. 6b). This self-assembly approach has the advantage that it is cost effective, reproducible and is easy to scale up. Furthermore, the dispersant layer thickness can be controlled by the spacer configuration, dispersant packing density and $M_{\mathrm{w}}$. A particular advantage of the "grafting to" approach is that the density of one or multiple surface-presented (bio)ligands can be tailored by co-adsorbing differently functionalized dispersants in one or several subsequent assembly steps. ${ }^{22,63}$ The polymer grafting density achieved using the "grafting to" approach has on flat surfaces been shown to be significantly lower than for the "grafting from" approach. ${ }^{62}$ This difference in polymer grafting density is a result of the steric repulsion of adjacent polymer chains that defines the density 

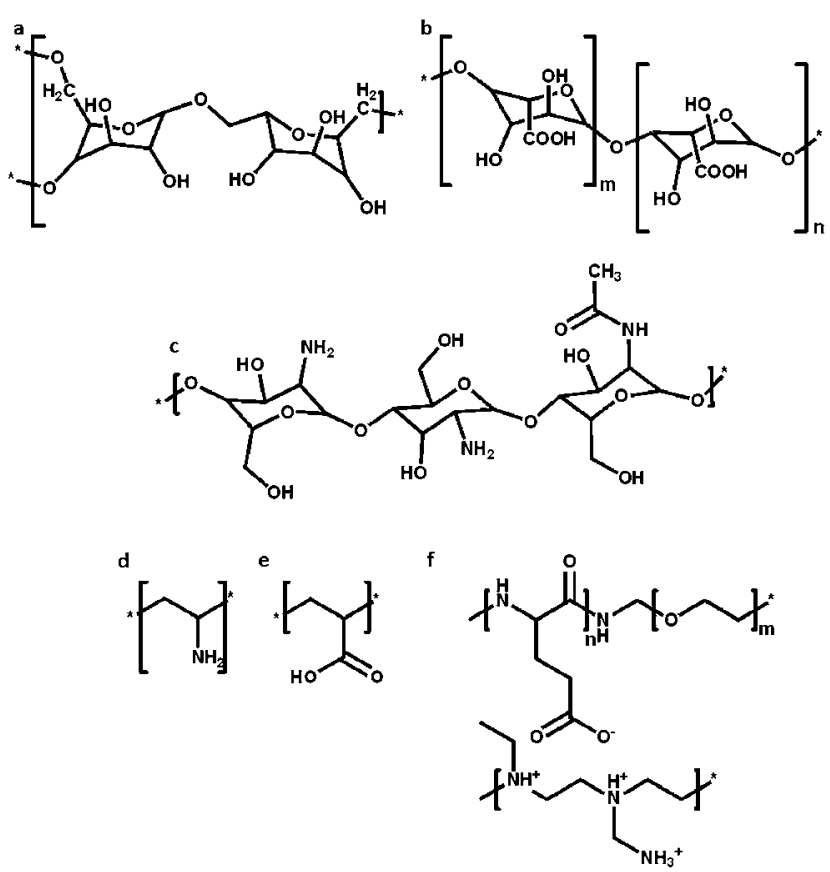

Fig. 4 Weakly adhering high $M_{\mathrm{w}}$ dispersants. Dispersants frequently used to coat superparamagnetic iron oxide NPs intended for biomedical applications are: (a) dextran, (b) alginate, (c) chitosan, (d) poly(vinyl amine), (e) poly(acrylic acid) and (f) polyelectrolytes such as poly (ethylene imine) (PEI) which electrostatically adsorbs on iron oxide NP surfaces. Further block-co-polymers such as poly(ethylene oxide)-poly (glutamic acid) can optionally be electrostatically bound to the PEI shell.

achievable for the grafting to approach, but not limiting polymers grown in situ on the surface using grafting from strategies. ${ }^{64,65}$ The rapidly increasing free volume available to dispersants on NPs with increasing distance from the core surface results in a lower steric repulsion of adjacent dispersants. Therefore, the difference in grafting density between surfaces
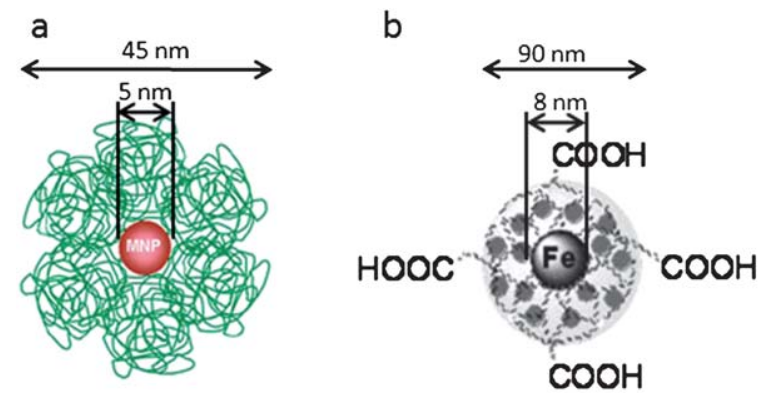

Fig. 5 Superparamagnetic iron oxide NPs stabilized with weakly adhering high $M_{\mathrm{w}}$ dispersants. (a) Superparamagnetic iron oxide NPs were individually stabilized with dextran where dextran was crosslinked after adsorption on the iron oxide NP surface. Crosslinking of dextran was done to decrease the tendency of dextran to desorb from the iron oxide NP surface. ${ }^{208}$ (Reproduced by permission of The Royal Society of Chemistry.) (b) Superparamagnetic iron oxide NPs were coated with poly (acrylic acid) (PAA). ${ }^{209}$ (Copyright Wiley-VCH Verlag GmbH \& Co. $\mathrm{KGaA}$. Reproduced with permission.) In contrast to the cartoons, the physisorption of high $M_{\mathrm{w}}$ dispersants with low affinity to iron oxide causes enwrapment of multiple cores, leading to broadly distributed hydrodynamic diameters many times larger than the core diameter. modified through the "grafting from" and the "grafting to" approach is expected to decrease with increasing surface curvature (Fig. 7). However, it should persist and limit the maximum $M_{\text {w }}$ of dispersants that can be used to form a complete shell around the core with the grafting to method.

\subsection{Anchors}

Irrespective of whether low $M_{\mathrm{w}}$ dispersants are grafted to or from the surface, they have to firmly adhere to the NP surface through suitable anchors (Fig. 2b). Ideally, the binding affinity of anchors is high and the desorption rate $k_{\text {off }}$ low so that anchors can irreversibly bind spacers to uncoated NPs and are able to replace hydrophobic capping agents such as oleic acid often used to synthesize monodisperse superparamagnetic iron oxide NPs. ${ }^{26}$ Naturally, anchors that meet these stringent requirements can be used both to immobilize initiators on a surface to graft dispersants from NP surfaces and to graft polymer brushes to NP surfaces. Typical anchors to surface modify superparamagnetic iron oxide NPs described in the literature are catechols ${ }^{66-69}$ or catechol derivatives, ${ }^{22,23,70,71}$ carboxy groups, ${ }^{72-74}$ phosphonates ${ }^{73,75,76}$ and silanes. ${ }^{77-81}$ Superparamagnetic iron oxide NPs individually stabilized with these dispersants are increasingly used for numerous biomedical applications (Table 1 and Fig. 8). Despite the central importance to define the stability of the NP shell and to define the density of functional units on a NP, the influence of different anchors on iron oxide NP stability was only recently systematically studied. Catechol derivatives with large variations in affinity to iron oxide were investigated as anchors for low $M_{\mathrm{w}}$ PEG based dispersants to stabilize superparamagnetic iron oxide NPs. ${ }^{23}$ Thorough characterization of iron oxide NP stability and its relation to successful in vivo application as a function of choice of anchor thus remains scant.

As a consequence of the lack of characterization of anchor stability, only few irreversibly binding anchors are known. Therefore, the main part of the literature on MR contrast agents deals with investigations on shells consisting of reversibly binding dispersants. One remedy to this which has been tried is immobilization through multiple reversibly adsorbing anchors. ${ }^{82}$ Multiple anchors per dispersant decrease the desorption rate of polymers ${ }^{82}$ provided they are interconnected by flexible linkers. Flexible interconnecting linkers between anchors are required for simultaneous binding of anchors also to the highly curved surface of a NP. However, the area multiple anchors occupy is considerably larger than that of a single anchor. Thus, multiple anchors might critically decrease the dispersant packing density on highly curved surfaces where steric repulsion of adjacent spacers is greatly reduced and therefore dispersant packing density must be increased to densities approaching that given by the individual anchors. ${ }^{83}$

Among the commonly described anchors phosphonic acid has been reported to be too weak to replace carboxy groups from iron oxide NP surfaces. ${ }^{84}$ Furthermore, carboxy groups were shown to be replaced by proteins ${ }^{85}$ and performed unfavorably as anchors to stabilize superparamagnetic iron oxide NPs compared to catechols and nitrocatechols. ${ }^{23}$ Silanes pose experimental difficulties in the assembly of dispersants on the iron oxide NP surface as they have to be adsorbed in water-free solvents and 
a
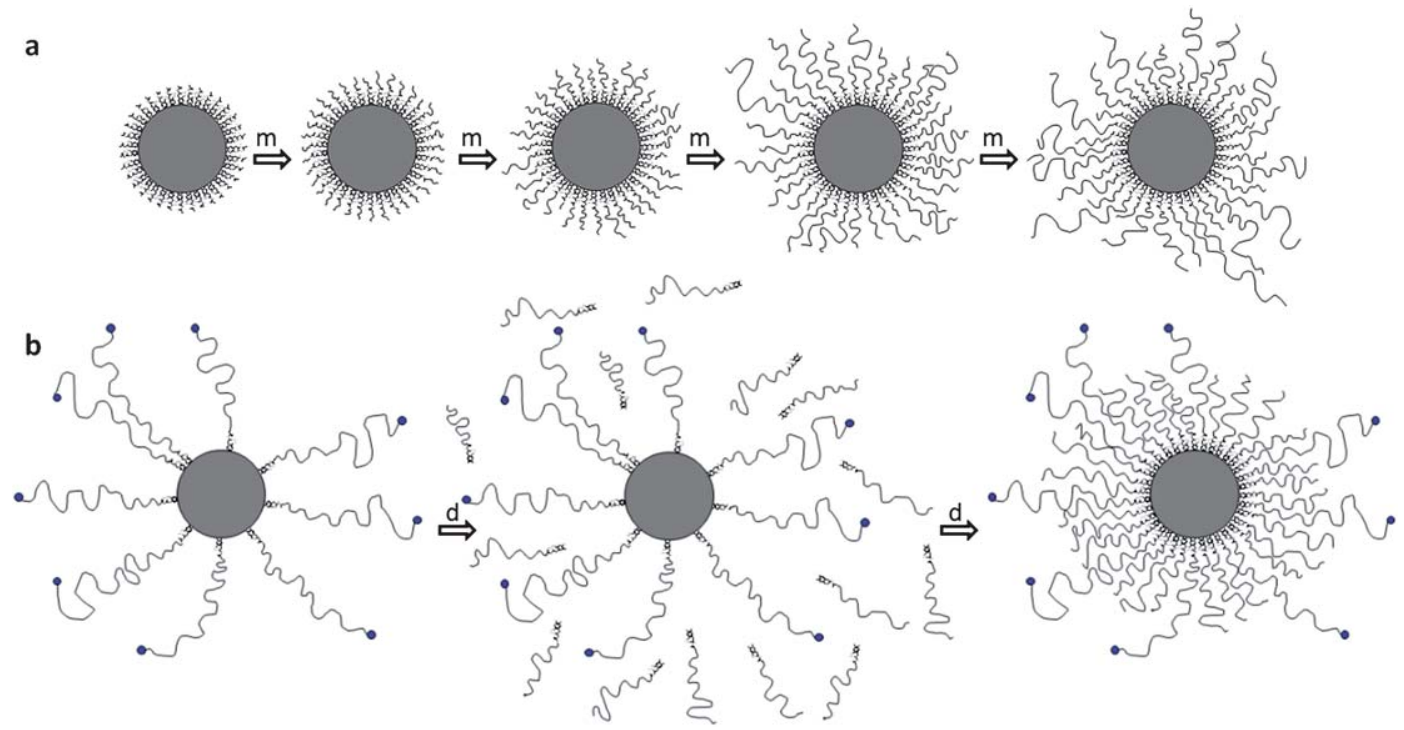

Fig. 6 Modifying iron oxide NP surfaces with low $M_{\mathrm{w}}$ dispersants. Low $M_{\mathrm{w}}$ dispersants can be (a) grafted from and (b) grafted to the NP surface. If low $M_{\mathrm{w}}$ dispersants are grafted from the NP surfaces, initiators are firmly bound to the NP surface. After monomers (m) were added to the NP dispersion dispersants are grown in situ, typically through a radically initiated chemical reaction. Dispersants grown in situ are densely packed on the NP surface, however, control over dispersant length, polydispersity and density of functionalities presented at the NP surface is difficult. Alternatively, dispersants (d) are synthesized prior to the adsorption on the NP surface. These dispersants are grafted to the NP surface. The resulting dispersant packing density is below that of dispersants grafted from the NP surface. However the grafting to approach allows for close control over the dispersant shell thickness and density of functionalities presented at the NP surface. The latter is achieved by adsorbing a defined concentration of functionalized dispersants at the NP surface. The NP surface is subsequently back-filled with non-functionalized dispersants.

can crosslink, which compromises control over the assembly of silane anchored dispersants. ${ }^{86}$

In recent years, inspired by the presence of catechols in organisms for fixation of metals and for surface adhesion, superparamagnetic iron oxide NPs have increasingly been surface modified using this chemical motif (Table 1) ${ }^{87}$ Because of the biological relevance of DOPA/ $\mathrm{Fe}^{3+}$ and dopamine/ $\mathrm{Fe}^{3+}$ complexes, their structure ${ }^{88-90}$ and electronic interactions ${ }^{91,92}$ have been studied in detail. Furthermore, the crystallographic and electronic structure of peptides, ${ }^{93}$ proteins ${ }^{94}$ and models for catechol dioxygenases complexed with iron ions ${ }^{89,95-97}$ have been reported. Despite their popularity as anchors fueled by biological inspiration and initial investigations on their application as dispersant anchors for iron oxide NPs, the suitability of catechols as anchors to stabilize iron oxide NPs is debated. Iron is well known to catalyze catechol oxidation leading to semiquinones, quinones and eventually carboxy-containing products. ${ }^{98-100}$ In line therewith, oxidative degeneration of dopamine adsorbed on iron oxide NPs resulting in a loss of NP stability was reported. ${ }^{101}$ Recently, cryo-TEM images of PEG-dopamine stabilized $\mathrm{Fe}_{3} \mathrm{O}_{4}$ NPs revealed NP agglomeration. ${ }^{102}$ These reports question the suitability of dopamine as an anchor for steric stabilization of superparamagnetic iron oxide NPs. Also surface corrosion of iron oxide NPs as a result of the replacement of oleic acid by dopamine has been reported, ${ }^{66}$ but is unlikely given the weak affinity of dopamine to surface presented iron ions. ${ }^{23,103}$

Already in 1976 it was noticed that electronegative substituents strengthen the iron-catechol bond to an extent where bonds between nitro-substituted catechols (so-called nitrocatechols) and iron remained unchanged for $24 \mathrm{~h}$ at $25{ }^{\circ} \mathrm{C}$ which was considered to be an irreversible bond. ${ }^{104}$ It was speculated that nitrocatechols can act as oxidizing agents which was assumed to be the reason for this exceptionally strong bond. ${ }^{104}$ Detailed studies on the binding of nitrocatechols to iron ions revealed a significantly lower tendency to generate radicals for nitrocatechol/iron compared to catechol/iron complexes. ${ }^{105}$ Based on complexation studies of these anchors with $\mathrm{Fe}^{3+}$, the increased complexation strength of electronegatively substituted catechols compared to unsubstituted counterparts was related to the increased acidity of the former compounds. ${ }^{106,107}$ Recently, EPR studies on superparamagnetic iron oxide NPs coated with nitroDOPA revealed an enhanced electron density at

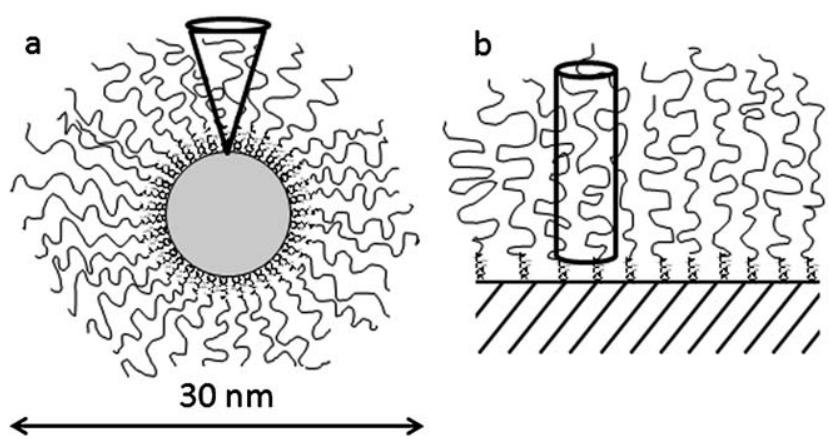

Fig. 7 Free volume of dispersants. The free volume of dispersants adsorbed on (a) NPs and (b) flat surfaces. While the free volume of dispersants adsorbed on NPs increases conically with increasing distance to the NP surface, it remains constant for dispersants adsorbed on planar surfaces. 


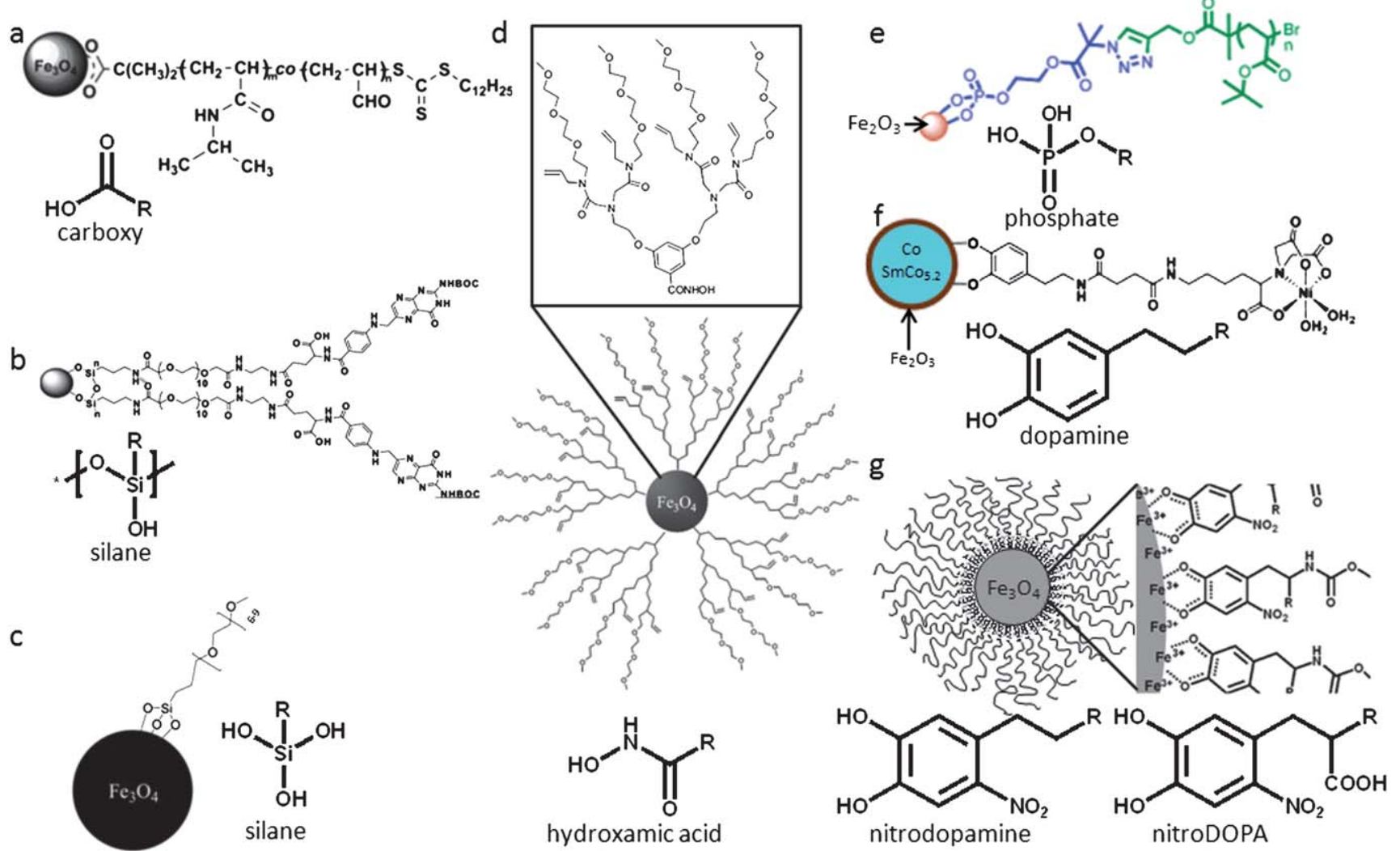

Fig. 8 Anchors for low $M_{\mathrm{w}}$ dispersants. Some of the more frequently used and recently described anchors to sterically stabilize superparamagnetic iron oxide NPs with low $M_{\mathrm{w}}$ dispersants are (a) carboxy groups ${ }^{210}$ (Journal of polymer science. Part A, Polymer chemistry by JOHN WILEY \& SONS, INC. Reproduced with permission of JOHN WILEY \& SONS, INC. in the format Journal via Copyright Clearance Center.), (b) crosslinked slianes ${ }^{211}$ (Journal of the American Chemical Society by American Chemical Society. Reproduced with permission of AMERICAN CHEMICAL SOCIETY in the format Journal via Copyright Clearance Center.), (c) silanes ${ }^{212}$ (ACS nano by American Chemical Society. Reproduced with permission of AMERICAN CHEMICAL SOCIETY in the format Journal via Copyright Clearance Center.), (d) hydroxymic acid ${ }^{213}$ (Copyright Wiley-VCH Verlag $\mathrm{GmbH} \&$ Co. KGaA. Reproduced with permission.), (e) phosphonates ${ }^{214}$ (Journal of the American Chemical Society by American Chemical Society. Reproduced with permission of AMERICAN CHEMICAL SOCIETY in the format Journal via Copyright Clearance Center.), (f) catechols such as dopamine $^{215}$ (Journal of the American Chemical Society by American Chemical Society. Reproduced with permission of AMERICAN CHEMICAL SOCIETY in the format Journal via Copyright Clearance Center.) and (g) electronegatively substituted catechols like nitrodopamine and nitroDOPA.

nitroDOPA anchors and electron depleted iron ions on the NP surface upon adsorption on iron oxide NPs. ${ }^{108,109}$ Dopamine is instead known to be oxidized if adsorbed on iron oxide surfaces. ${ }^{101}$

The strong complexation of nitrocatechols to $\mathrm{Fe}^{3+}$ ions and enhanced electron density at the nitroDOPA anchors have been shown to directly relate to the high stability of grafted polymer films when nitrocatechols are used as anchors. The stability of superparamagnetic iron oxide NPs with shells of PEG-nitroDOPA or PEG-nitrodopamine was shown to be much higher than that of iron oxide NPs stabilized with PEG-DOPA and PEG-dopamine. ${ }^{23}$ Follow-up studies revealed that the binding affinity of anchors to the metal ion of oxides has to be optimized rather than maximized to achieve good iron oxide NP stability, ${ }^{108,109}$ since the anchor with the highest binding affinity resulted in dissolution of the iron oxide cores. Furthermore, these studies also indicated that knowledge acquired on anchor/metal ion complexes ${ }^{108,109}$ and flat surfaces ${ }^{23}$ can be translated into the suitability of anchors to surface-modify respective oxide NPs. More generally, electronegatively substituted catechols such as nitrocatechols ${ }^{110,111}$ and chlorocatechols ${ }^{111,112}$ were reported not only to bind well to iron oxide surfaces but also to $\mathrm{Al}_{2} \mathrm{O}_{3}$ and $\mathrm{TiO}_{2}$ surfaces, and were used to form polymer brushes on planar surfaces of the latter. ${ }^{113}$

\subsection{Spacers}

Apart from anchors, suitable spacers are crucial to achieve good steric NP stability (Fig. 2b). Spacers have to prevent that NPs get into too close proximity, where van der Waals and magnetic attraction interactions start to dominate. If two sterically stabilized cores approach each other, the volumes of the respective polymer shells are confined. This reduces the entropy of dispersants and increases the osmotic pressure between NPs. The resulting repulsive potential critically depends on the dispersant density profile, packing density, ${ }^{114}$ binding reversibility and solvent quality with respect to the dispersants. ${ }^{115}$ The design and optimization of sterically stabilized core-shell NPs would be greatly facilitated if these parameters were known. Only then can the inter-particle potentials be calculated. ${ }^{116,117}$ However, the 
literature on the dispersant density profile of core-shell NPs is inconclusive.

Polymer density profile on planar surfaces. Numerous theoretical $^{118-120}$ and experimental ${ }^{121}$ studies on the density profile of polymers adsorbed on planar surfaces have been published. Scaling theories applied e.g. by de Gennes resulted in a logarithmically decaying inter-particle potential. ${ }^{116}$ Self-consistent field (SCF) theory calculations were used to refine the dispersant density profile by relaxing the stringent requirement that chain ends are located at the interface resulting in a parabolic polymer density profile. ${ }^{119,120,122}$ Shim and Cates further refined this model by taking into account the finite extensibility of the polymer chains and extended it to high polymer coverages. ${ }^{123}$ Based on these refined models it was found that with increasing surface coverage, the density profile changes from a parabolic to a stepfunction profile for tightly packed, highly stretched polymers.

Polymer density profile on highly curved surfaces. The scaling theory applied to NPs resulted in a dispersant density profile decaying exponentially with increasing distance from the NP surface. ${ }^{124}$ Thus, this and follow-up studies showed that the density profile of dispersants obtained from scaling theories differs between NPs and flat surfaces. ${ }^{125}$ However, scaling theories and the Derjaguin approximation fail if the dispersant chain dimensions approach or exceed the core diameter, which is usually the case for NPs sterically stabilized in aqueous media. A different scaling behavior of chains grafted to NP surfaces was found by extending the SCF theory ${ }^{\mathbf{1 2 6}}$ and through Monte Carlo simulations. ${ }^{127}$ Detailed information about the dispersant density profile on NPs can only be extracted if more refined theories than the de Gennes theory such as the SCF theory are applied. ${ }^{128}$ Ball et al. who used the SCF theory ${ }^{129}$ and $\mathrm{Li}$ and Witten who compared the SCF theory to exact solutions obtained by minimizing the free energy ${ }^{130}$ found that in the limit of low dispersant packing densities and high dispersant $M_{\mathrm{w}} \mathrm{s}$, the density profile close to the NP interface is depleted from free polymer chain ends.

However, the assumption of negligible inter-chain penetration inherent to the models described above only holds for long chains and low packing densities. Short chains significantly interpenetrate adjacent chains, which alters the dispersant density profile. For polymer chains shorter than 1000 repeat units, deviations from the parabolic polymer density profile result in an earlier and gradual onset of repulsive inter-particle forces compared to interparticle potentials calculated for parabolic polymer density profiles. ${ }^{131}$ Furthermore, the dispersant density profile was predicted to undergo a smooth change from a parabolic decay at low surface curvatures and for small or stiff dispersants to a power law decay similar to that of star polymers if dispersants are adsorbed on highly curved surfaces or if dispersants are flexible. ${ }^{115,128}$

In summary, despite the numerous theoretical studies on the density profile of polymers adsorbed on highly curved surfaces and experimental investigations of the density profile of blockco-polymers ${ }^{132-134}$ the density profile of polymers adsorbed on highly curved surfaces is still debated. One of the main reasons for the inconclusive literature might be the lack of experimental data on dispersants irreversibly grafted to highly curved surfaces.
Reversible dispersant adsorption is likely to change the dispersant density profile as the latter is dependent on the dispersant packing density. Moreover, dispersant density profiles are often investigated with SANS. ${ }^{135}$ However, desorbed dispersants also scatter and therefore contribute to the total scattering curve measured for core-shell NPs. These desorbed dispersants thus hamper the analysis of scattering data. If dispersants were irreversibly bound to the NP surfaces through suitable anchors, the dispersant density profile could be assessed e.g. with SANS experiments.

\section{Effect of surface modification of nanoparticles on their biomedical performance protein resistance}

As mentioned above in the section on defining stability, superparamagnetic iron oxide NPs for most biomedical applications have to resist adsorption of biomacromolecules such as proteins in addition to avoiding aggregation. This property, which often is referred to as "stealth", is necessary for successful application in vivo. If plasma proteins such as opsonins are adsorbed on the NP surface, they induce NP uptake by macrophages, monocytes and dendritic cells and thus initiate NP clearance. ${ }^{136}$ Therefore, protein adsorption significantly decreases the circulation time of NPs. The requirement of resistance to protein adsorption precludes electrostatically stabilized NPs because most proteins express surface charges. This limits effective stabilization strategies to those using sterically stabilizing polymer shells, for which proteins and other biomolecules have no affinity for the spacer part of the shell.

The most commonly used dispersant spacer that renders NPs stealth is PEG. ${ }^{11,21}$ PEG-modified surfaces exhibit the smallest attractive van der Waals forces to proteins compared to other well-known water soluble polymers due to the low refractive index of PEG. ${ }^{137}$ Furthermore, it is thought that protein adsorption leads to confinement of the PEG chains resulting in an energetically unfavorable entropy decrease. ${ }^{138}$ Yet another factor contributing to the ability of PEG to render surfaces protein resistant is the ordering of water around PEG chains, ${ }^{139}$ which prevents direct contact of PEG with proteins. ${ }^{140}$ However, PEG is known to be prone to degeneration if applied in vivo. ${ }^{\mathbf{1 4 1 , 1 4 2}}$ In vitro studies showed that poly(2-methyl-2-oxazoline) (pMOXA) has similar protein repelling properties to PEG, but is less prone to degradation. ${ }^{143}$ Thus, pMOXA is a promising alternative to PEG for the spacer part of low $M_{\mathrm{w}}$ dispersants for in vivo applications.

Studies on the influence of shell thickness, dispersant packing density and dispersant conformation on the protein resistance of sterically stabilized NPs have to be designed carefully to make sure that the right conclusions are arrived at. It has to be ensured that the dispersants are irreversibly and homogeneously adsorbed on the NP surface, so that the exchange of dispersants by proteins can be excluded and the protein resistance unequivocally attributed to the PEG shell.

Protein adsorption has been studied in detail on PEG-modified planar surfaces where quantitative surface sensitive characterization techniques such as XPS and time-of-flight secondary ion mass spectroscopy (ToF-SIMS) are at hand. ${ }^{\mathbf{2 2 , 1 4 4 , 1 4 5}}$ It was found that protein adsorption decreases almost linearly with increasing ethylene glycol (EG) monomer surface density. If planar surfaces 
are coated with EG surface densities $>15-30 \mathrm{~nm}^{-2}$, they become protein resistant. ${ }^{\mathbf{8 2 , 1 4 4}}$

The existence of a threshold value for the EG density for protein resistance on surfaces has direct consequences on the PEG packing density. In fact, a high PEG packing density has been shown to be crucial to prevent adsorption especially of small proteins. The adsorption of large proteins on surfaces was shown less sensitive to the PEG packing density. ${ }^{145-148}$ Furthermore, a threshold value for the EG density also closely relates to the $M_{\mathrm{w}}$ required to render surfaces protein resistant. The higher the PEG $M_{\mathrm{w}}$, the lower the polymer packing density can be to impart complete protein resistance to PEGylated surfaces. Generally, protein resistance requires that PEG is in the brush regime where the distance between adjacent chains $D$ is smaller than the Flory radius $R_{\mathrm{F}}$ of the polymers. ${ }^{118,149}$

Similarly, the PEG $M_{\mathrm{w}}$ and the EG surface density were shown to be crucial parameters for the protein resistance and thus circulation time of NPs. The EG density on NPs might have to be higher than on planar surfaces to render NP surfaces protein resistant due to the high surface curvature of NPs that leads to a conically increasing free volume for dispersants starting from the NP surface (Fig. 7). However, the general principles for protein resistance described above are expected to hold irrespective of the surface curvature.

This was exemplified in a study, where protein resistance of $100 \mathrm{~nm}$ diameter poly(lactic acid) (PLA) NPs stabilized with PEG ( $2 \mathrm{kDa}$ ) could only be obtained if the PEG packing density was $\geq 0.2$ molecules $\mathrm{nm}^{-2}{ }^{150}$ For lower PEG packing densities, the circulation time increased with increasing PEG $M_{\mathrm{w}}$ s due to an increased EG density. ${ }^{56}$ Protein adsorption on $200 \mathrm{~nm}$ diameter PLA NPs could be significantly decreased if at least $5 \mathrm{wt}$ $\%$ PEG $(5 \mathrm{kDa})$ was added to the PLA NP surface. ${ }^{55}$ At this concentration, PEG should be in the brush regime as the distance between two PEG chains was $D=1.4 \mathrm{~nm}^{56}$ whereas $R_{\mathrm{F}}$ of PEG $(5 \mathrm{kDa})=5.1 \mathrm{~nm}$ and the curvature of the core is rather low. However, due to steric repulsion, the PEG packing density will also decrease on NP surfaces with increasing PEG $M_{\mathrm{w}}$. Too low PEG packing density in itself compromises NP stability and sets an upper limit to the suitable $M_{\mathrm{w}}$ range. ${ }^{41}$ This is a particular problem if the dispersant $M_{\mathrm{w}}$ is so high that only few polymer chains can attach during grafting or remain attached after hydration. Therefore, PEG $M_{\mathrm{w}} \mathrm{s}$ between 1.9 and $5 \mathrm{kDa}$ were found to be optimal to surface modify superparamagnetic iron oxide NPs intended for biomedical applications. ${ }^{7,146,151}$ NPs stabilized with PEGylated dispersants that fulfill these requirements of high dispersant packing densities and PEG $M_{\mathrm{w}} \mathrm{s}$ between $1.9 \mathrm{kDa}$ and $5 \mathrm{kDa}$ showed prolonged circulation times. ${ }^{152,153}$

\subsection{Biodistribution — relation to nanoparticle size and stability}

In addition to the surface chemistry that determines the affinity of proteins to adsorb on NP surfaces, NP size, shape, ${ }^{50,154}$ and surface charge ${ }^{155}$ also determine the fate of NPs in vivo. ${ }^{156}$ While particles larger than $200 \mathrm{~nm}$ are rapidly cleared by the spleen, NPs smaller than 10-50 $\mathrm{nm}$ are generally removed from the body through extravasion and renal clearance. ${ }^{11,58}$ The optimal hydrodynamic diameter range for in vivo applications of intravenously injected NPs that require prolonged blood half-life times is therefore $10-100 \mathrm{~nm}$. The hydrodynamic diameter range together with the range of dispersant shell thicknesses that lead to good NP stabilities described above limit the respective core and spacer sizes of NPs.

PEG stabilized superparamagnetic iron oxide NPs with a hydrodynamic diameter between 30 and $50 \mathrm{~nm}$ have been reported to mainly end up in the liver and spleen if administered in nude mice. ${ }^{157}$ Their concentration in the liver and spleen decreased to $40 \%$ and $20 \%$, respectively, of the injected dose within 72 h. ${ }^{157}$ However, the clearance of PEGylated NPs was shown to depend on the affinity of dispersant anchors to the NP surface. ${ }^{136,158}$ If PEG was covalently attached to the surface, the NP circulation time was substantially prolonged compared to that of NPs where PEG was physisorbed to the surface. ${ }^{56,158}$ The fast clearance of the latter NPs was assigned to the fact that proteins could replace physisorbed PEG.

Similar to PEG stabilized superparamagnetic iron oxide NPs, dextran coated, agglomerated iron oxide NPs accumulated in the liver and spleen. In addition to slow clearance and a tendency to agglomerate, ${ }^{159}$ dextran coated NPs have also been shown to induce differentiation of monocytes into macrophages. ${ }^{55}$

\section{Effect of nanoparticle stability on magnetic properties}

One essential parameter that describes the magnetic response of superparamagnetic iron oxide NPs is their saturation magnetization $\left(M_{\mathrm{s}}\right)$. The higher the $M_{\mathrm{s}}$ of superparamagnetic iron oxide NPs, the easier they can be magnetically separated and ferried to desired locations. Furthermore, a high $M_{\mathrm{s}}$ of superparamagnetic iron oxide NPs locally induces higher magnetic field gradients if dispersed in solutions and subjected to an external homogeneous magnetic field. These magnetic field perturbations are responsible for changed relaxivities $r_{2}$ of water molecules measured in MRI. Thus, the higher the $M_{\mathrm{s}}$ of superparamagnetic iron oxide NPs with everything else being equal, the more effective they are as MR contrast agents. ${ }^{11}$

The magnetic properties of NPs depend on the composition, size and shape of the NP core. ${ }^{11}$ However, $M_{\mathrm{S}}$ of superparamagnetic iron oxide NPs is always below that of the respective bulk materials and decreases with decreasing core size. ${ }^{160}$ This decrease was assigned to surface anisotropy effects $^{161,162}$ that become increasingly important with increasing surface to volume ratio and thus with decreasing NP size.

\subsection{Effect of surface modification on saturation magnetization of superparamagnetic iron oxide nanoparticles}

The $M_{\mathrm{s}}$ has been shown to decrease if superparamagnetic iron oxide NPs are sterically stabilized. ${ }^{22,163}$ However, to directly compare $M_{\mathrm{s}}$ values of stabilized and unstabilized superparamagnetic iron oxide NPs, the $M_{\mathrm{s}}$ has to be normalized to the mass of iron oxide to account for the lower $\mathrm{wt} \%$ of NP cores in sterically stabilized compared to bare iron oxide NPs. The lower $M_{\mathrm{s}}$ of sterically stabilized superparamagnetic iron oxide NPs likely is related to interactions of anchors with surface bound iron ions that influence the magnetic properties of the iron oxide NP surface layer. ${ }^{108,109}$ Therefore, iron oxide NP stability and size have to be traded off against high $M_{\mathrm{s}}$ values. 
One common way in the literature to demonstrate good magnetic properties of stabilized superparamagnetic iron oxide NPs is to show their attraction in solution to a small tabletop magnet. However, individually stabilized superparamagnetic iron oxide NPs have too low $M_{\mathrm{s}}$ to be strongly attracted by a small tabletop magnet. In contrast, agglomerates are readily attracted by such magnets. ${ }^{22,39}$ Therefore, tests in which iron oxide NPs are rapidly cleared from solutions using tabletop magnets not only indicate good magnetization of the superparamagnetic iron oxide NPs but also poor NP stability.

\subsection{Relaxivity}

Superparamagnetic iron oxide NPs enhance contrast in MR images by changing the relaxation times $r_{1}$ and $r_{2}$ of adjacent water molecules. ${ }^{11} r_{1}$ is mainly determined by the exchange rate of water molecules in the first hydration shell of superparamagnetic iron oxide NPs. Thus, it depends on the accessibility of water molecules to the iron oxide core surface. Commercially available superparamagnetic iron oxide NPs are coated with the reversibly adsorbing dextran that allows water to readily exchange also in close proximity to the NP surface (Fig. 2a and 5). However, if superparamagnetic iron oxide NPs are stabilized with low $M_{\mathrm{w}}$ dispersants through irreversibly binding anchors and at high dispersant packing densities, the accessibility of water molecules to the iron oxide surface is reduced. Therefore, $r_{1}$ contrast of iron oxide cores individually stabilized with low $M_{\mathrm{w}}$ dispersants that are irreversibly adsorbed at the iron oxide NP surface at high packing densities is lower ${ }^{41}$ compared to dextran coated iron oxide NPs.

Table 2 provides an overview of relaxivities of some commercially available superparamagnetic iron oxide NPs compared to individually stabilized iron oxide NPs. ${ }^{13,16}$ It is well known that $r_{2}$ increases with increasing iron oxide NP size. ${ }^{164,165}$ Furthermore, iron oxide NP agglomeration ${ }^{166,167}$ and controlled crosslinking of iron oxide $\mathrm{NPs}^{168}$ have also been shown to increase $r_{2}$. This was confirmed by Monte Carlo simulations. ${ }^{169,170}$ Therefore, $r_{2}$ of individually stabilized superparamagnetic iron oxide NPs is lower compared to that of commercially available, dextran coated, agglomerated NPs (Table 2). However, low $M_{\mathrm{w}}$ dispersants that are firmly bound to the iron oxide NP surface through suitable anchors allow independent tuning of the core diameter and shell thickness. This opens up the possibility to increase $r_{2}$ without sacrificing iron oxide NP stability, by increasing core size up to the limit where iron oxide NPs become ferromagnetic. Therefore, individually stabilized superparamagnetic iron oxide $\mathrm{NPs}^{41}$ can have $r_{2}$ values comparable to those of commercially available iron oxide based MR contrast agents, ${ }^{13,16}$ since the former have a hydrodynamic diameter many times smaller than the commercially available analogues (Table 2).

The influence of the dispersant shell on the relaxivity is still debated. ${ }^{171,172} r_{2}$ values reported by Duan et al. were claimed to depend on the hydrophilicity of the polymer shell. ${ }^{172}$ However, relaxivity did not change systematically with the hydrodynamic diameter of superparamagnetic iron oxide NPs. ${ }^{172}$ In contrast, LaConte et al. reported a decrease in $r_{2}$ with increasing hydrodynamic diameter for PEG with $M_{\mathrm{w}}<1 \mathrm{kDa} .{ }^{171}$ Iron oxide NPs stabilized with PEGs with $M_{\mathrm{w}} \mathrm{s}$ between 1 and $5 \mathrm{kDa}$ had within experimental error the same relaxivities. However, it is questionable, whether PEG spacers with $M_{\mathrm{w}}<1 \mathrm{kDa}$ result in stable NPs. Thus, the dependence of $r_{2}$ on the dispersant shell thickness likely is related to the limited stability of NPs coated with PEGs that have $M_{\mathrm{w}}<1 \mathrm{kDa}$ rather than to the fact that the PEG shell slows down the water exchange rate.

\subsection{Specific adsorption rate (SAR)}

The specific adsorption rate (SAR) determines how effectively NPs generate heat if they are exposed to an alternating magnetic field (AMF). The SAR is the most important property for the use of superparamagnetic iron oxide NPs in hyperthermia treatment or for triggering release of cargo encapsulated in thermoresponsive drug delivery vehicles. ${ }^{58}$

For superparamagnetic NPs that have small magnetic anisotropies, the SAR at a fixed frequency $\nu$ is proportional to the relaxation time $\tau$ of NPs. ${ }^{58} \tau$ increases with increasing core size of the NPs. ${ }^{173}$ Therefore, the SAR increases with increasing NP size up to a critical core diameter $d_{\text {crit }}$. If $\tau>(2 \pi \nu)^{-1}$, the Néel and/or Brownian relaxations of NPs cannot follow the alternating magnetic field and thus the SAR then rapidly decays with increasing $\tau$ and therefore NP size. ${ }^{58,173}$

Iron oxide NP agglomeration is known to increase interparticle interactions ${ }^{174}$ which decreases Néel relaxation losses at frequencies typically used for SAR applications. ${ }^{175}$ Thus, steric stabilization of individual iron oxide cores increases the SAR as was experimentally shown on agglomerated and with poly (methyl methacrylate) (p(MMA)) stabilized superparamagnetic iron oxide NPs. ${ }^{175}$

Table 2 Comparison of relaxivities of commercially available superparamagnetic iron oxide NPs stabilized with physisorbed dispersants to selected individually stabilized superparamagnetic iron oxide NPs with defined core sizes

\begin{tabular}{|c|c|c|c|c|c|c|c|}
\hline Trade name & $d_{\text {core }} / \mathrm{nm}$ & $d_{\text {hydrodynamic }} / \mathrm{nm}$ & Dispersant & $r_{1} / \mathrm{mM}_{\mathrm{Fe}} \mathrm{s}^{-1}$ & $r_{2} / \mathrm{mM}_{\mathrm{Fe}} \mathrm{s}^{-1}$ & $r_{2} / r_{1}$ & Ref. \\
\hline Resovist & 4.2 & 62 & Carboxydextran & $24-25$ & $107-151$ & $4.5-6$ & 255,256 \\
\hline $\begin{array}{l}\text { Individually } \\
\text { stabilized iron oxide NPs }\end{array}$ & 5 & 26 & PEG(5)-nitroDOPA & 12 & 82 & 6.8 & 258 \\
\hline
\end{tabular}




\section{Iron oxide nanoparticle functionalization}

\subsection{Surface presentation of targeting ligands}

Different types of unfunctionalized superparamagnetic iron oxide NPs stabilized with weakly adsorbed high $M_{\mathrm{w}}$ dispersants, such as dextran, are FDA approved. ${ }^{176,177}$ They are used in clinics as negative MR contrast agents to detect lesions mainly in the liver and spleen. ${ }^{58,176} \mathrm{~A}$ comprehensive list of non-targeted commercially available and in research labs developed targeted iron oxide based MR contrast agents, was recently published by Laurent et al. ${ }^{58}$

Dispersants adsorbed on NP surfaces allow for functionalization of NPs, an option that becomes increasingly important for many applications especially in the biomedical field. ${ }^{6,58,178}$ Potential targeting moieties are antibodies, ${ }^{179}$ peptides, ${ }^{\mathbf{8 0}, 180-182}$ aptamers, ${ }^{183}$ DNA $^{168,184}$ and RNA $^{185}$ sequences. They are thought to be able to specifically bind appropriately functionalized NPs to desired locations.

Reports where in vivo targeting of superparamagnetic iron oxide NPs is claimed are numerous. However, the vast majority of iron oxide NPs are targeted towards the liver, kidney or lymph nodes, locations they naturally end up during clearance. ${ }^{186}$ Alternatively, iron oxide NPs were targeted to tumors and cancer cells, where they naturally accumulate due to the enhanced permeation retention (EPR) effect (Table 3).$^{\mathbf{5 8 , 1 8 7}}$ Proving specific targeting to such organs is therefore difficult as increased accumulation can occur also without specific binding to a target. Table 3 provides an overview of superparamagnetic iron oxide NPs individually stabilized with low $M_{\mathrm{w}}$ dispersants and selected examples of iron oxide NPs coated with dextran that were subsequently functionalized with ligands covalently bound to the dextran shell.

Key to successful targeting of NPs just as for successful stabilization is that functionalities are irreversibly bound to the NP surface. Only then can desorption of functional units from NP surfaces be prevented. Desorbed targeting ligands can block receptors before functionalized NPs reach these locations and the loss of ligands results in lower affinity of these NPs to the targeting sites. Irreversible binding of functionalities to NP cores can be fulfilled if spacers and functionalities are bound to NP surfaces through suitable anchors. If NPs are stabilized with low $M_{\mathrm{w}}$ dispersants, the ligand density presented at the NP surface can be closely controlled by co-adsorbing functionalized and unfunctionalized dispersants to the NP surface at defined molar ratios. ${ }^{22}$ This is in stark contrast to NP surfaces coated with physisorbed high $M_{\mathrm{w}}$ dispersants where the serpentine, constantly changing conformation prevents efficient addition and controlled presentation of ligands at the NP interface. ${ }^{188}$

One of the experimentally easiest and most versatile ways to functionalize NPs is the avidin-biotin coupling strategy. The avidin-biotin bond is one of the strongest non-covalent bonds, with a dissociation constant of $K_{\mathrm{d}} \approx 10^{-15} \mathrm{M} \cdot{ }^{189,190}$ For practical purposes, this is an irreversible bond, at least in the absence of external forces. Because many ligands are commercially available in a biotinylated form, this functionalization method is often used for research purposes. 22,191,192

Individually stabilized NPs functionalized through the biotinavidin coupling strategy possess a layer-by-layer build-up. The different layers are modularly interchangeable and allow to firmly attach active ligands to NP cores. One of the main disadvantages of NP functionalization through the biotin-avidin coupling strategy is the risk that NPs agglomerate during the layer-by-layer assembly and subsequent purification of functionalized NPs. Several different avidin derivatives are commercially available of which the least non-specifically interacting is neutravidin, but they all possess multiple binding sites for biotin. ${ }^{190}$ During and after biotinylated NPs are coated with neutravidin, more than one biotinylated NP can therefore bind to the same neutravidin leading to agglomeration (Fig. 9a). To prevent crosslinking during coating of biotinylated NPs with neutravidin, biotinylated NPs have to be slowly added to a solution containing an excessive amount of neutravidin, followed by thorough washing to remove excessive neutravidin. The resulting NPs have to be surrounded by a complete neutravidin monolayer. ${ }^{22}$

Neutravidin coated NPs furthermore bear the risk to crosslink while they are incubated with biotinylated ligands with multiple biotin sites per molecule (Fig. 9b). Large ligands such as antibodies always present multiple biotin sites per molecule unless they are specifically engineered. Since such ligands are also expensive they cannot be dispersed at high concentrations to reduce the risk of crosslinking by following the strategy developed for the neutravidin functionalization step (Fig. 9b). Thus, the likelihood that biotinylated NPs crosslink during or after functionalization with biotinylated ligands though an avidin intermediate layer is high. Similar things were reported for biotinylated liposomes that were functionalized with biotinylated antibodies. ${ }^{193,194}$

Furthermore, for in vivo applications, functionalized NP dispersions typically have to be up-concentrated many times. This adds an additional preparation step and increases the risk for NP crosslinking. Needless to say that if dispersants are reversibly bound to the NP surface, dissociation of biotinylated dispersants that are linked to neutravidins also results in free neutravidin in solution which can be another cause for agglomeration and reduced targeting efficiency. Thus, while NP functionalization through the avidin-biotin coupling strategy is experimentally easy and versatile, it is only suited for applications that do not require long term NP stability and where NPs are applied under dilute conditions. As a neutravidin coating is likely to reduce the stealth property of NPs, neutravidin coated NPs are mainly suitable for in vitro applications where NPs do not have to be stealth.

The loss of stealth properties is minimized if the number of proteins in the ligand shell is minimized. This can be achieved by covalently linking ligands directly to the stealth PEG dispersant shell at a controlled density instead of using an avidin intermediate layer (Fig. 10). In the former case, specific and non-specific protein adsorptions to NPs are assumed to be limited to the added ligands. Superparamagnetic iron oxide NPs functionalized with covalently bound ligands have so far typically been coated with dispersants such as dextran that lack well defined anchors (Table 3). ${ }^{179}$ They therefore make a controlled surface presentation of functionalities difficult. ${ }^{\mathbf{1 8 2}}$

In contrast, superparamagnetic iron oxide NPs that are stabilized with low $M_{\mathrm{w}}$ dispersants, such as PEG-silanes, allow controlled presentation of ligands at the NP interface. This was 


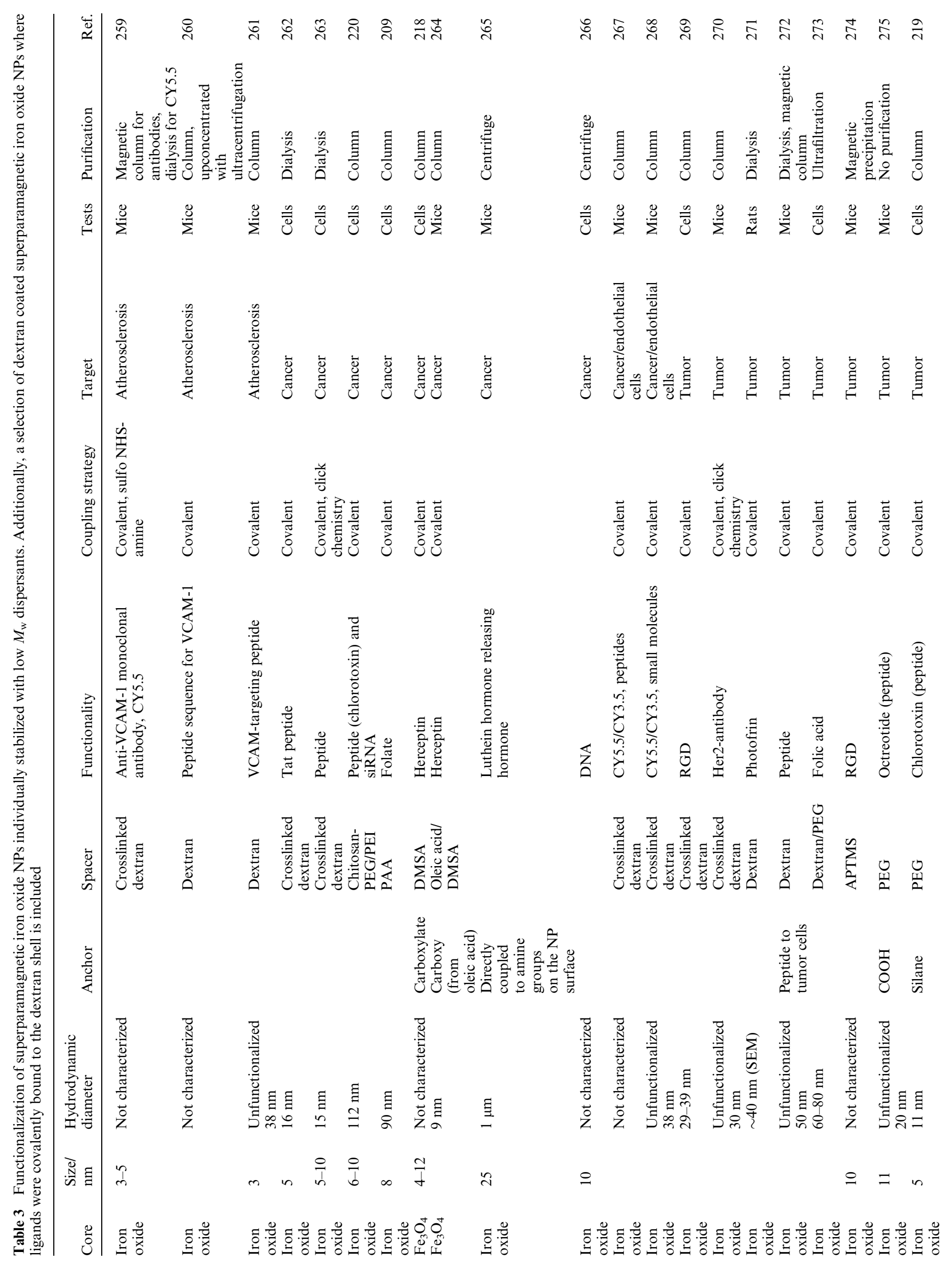



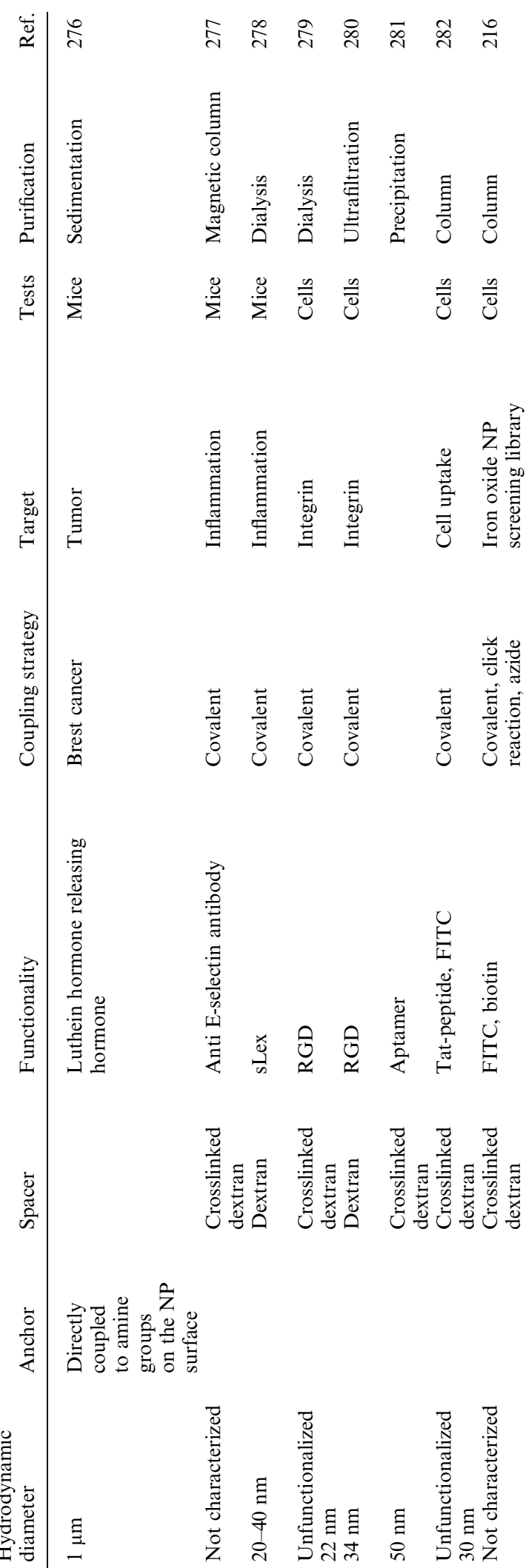

$$
\begin{aligned}
& 0 \\
& \stackrel{0}{n}
\end{aligned}
$$

demonstrated e.g. on chlorotoxin functionalized iron oxide NPs. ${ }^{9,78}$ Their performance and uptake was subsequently studied in vitro in cell assays. ${ }^{9,80}$ A controlled surface presentation of ligands is thought to increase the targeting efficiency by decreasing the risk that ligands are buried in the dispersant shell. Furthermore, it allows for a closer control over changes in the hydrodynamic diameter of NPs upon functionalization (Fig. 11) and enables optimizing the number of ligands bound to one NP to ensure sufficient binding affinity while minimizing non-specific interactions.

Because the hydrodynamic size of superparamagnetic iron oxide NPs significantly influences NP uptake by cells, ${ }^{195}$ control over the hydrodynamic diameter of NPs upon functionalization is crucial. Ligands, such as antibodies and peptides, are often comparable in size to the iron oxide $\mathrm{NPs}^{185}$ and their coupling can significantly change the hydrodynamic size (Fig. 11). Analogous to the case described for biotin-avidin functionalization above, ligands can also crosslink and cluster individually stabilized NPs if they bear multiple chemically reactive groups per NP (Fig. 11c). This significantly increases the hydrodynamic diameter. It prevents elucidation of the effect of ligands on the biodistribution and clearance of functionalized NPs as this is always convoluted with the increase in NP size. Therefore, it is of highest importance to measure the hydrodynamic diameter and $\zeta$ potential of NPs before and after ligands are coupled to their shells. Only if the NP size and surface charge are not significantly altered by the attached ligands can differences in the NP biodistribution and clearance rate unequivocally be attributed to a targeting effect. However, the hydrodynamic size of superparamagnetic iron oxide NPs after ligands were coupled to their shells is often not reported (Table 3). A few in vitro and in vivo studies where this has been done are described below.

The hydrodynamic size and $\zeta$ potential of iron oxide NPs functionalized with chlorotoxin ${ }^{185}$ and changes in the hydrodynamic size and surface charge upon assembling cRGD functionalized iron oxide NPs ${ }^{196}$ were reported. Preferred binding of cRGD functionalized over non-functionalized iron oxide NPs towards cancer cells was shown in in vitro studies. Because of similar values of the $\zeta$ potential and hydrodynamic size of unfunctionalized and cRGD functionalized iron oxide NPs, the preferred binding of the latter NPs towards cancer cells can be assigned to specific binding of these NPs.

An alternative way to elucidate binding specificity of NPs is to functionalize them with different peptide sequences of similar size and charges. A comparison of the binding of NPs functionalized e.g. with native and scrambled RGD sequences to cells allows us to assess the binding specificity of such NPs. ${ }^{180}$

Superparamagnetic iron oxide NPs have most frequently been functionalized with peptides (Table 3). The increasing number of reports on peptide functionalized iron oxide NPs might be related to the feasibility to assemble NPs that have a high affinity towards receptors in a controlled way, thanks to the smaller size and that peptides can be designed and chosen to have only a few coupling sites per molecule. For steric reasons, the large size of antibodies only allows a limited number to be attached to the NP surface. However, the binding affinity of functionalized NPs was greatly enhanced if NPs could bind to receptors through the attachment of multiple ligands compared to NPs functionalized with a low ligand density where statistically only one ligand per 


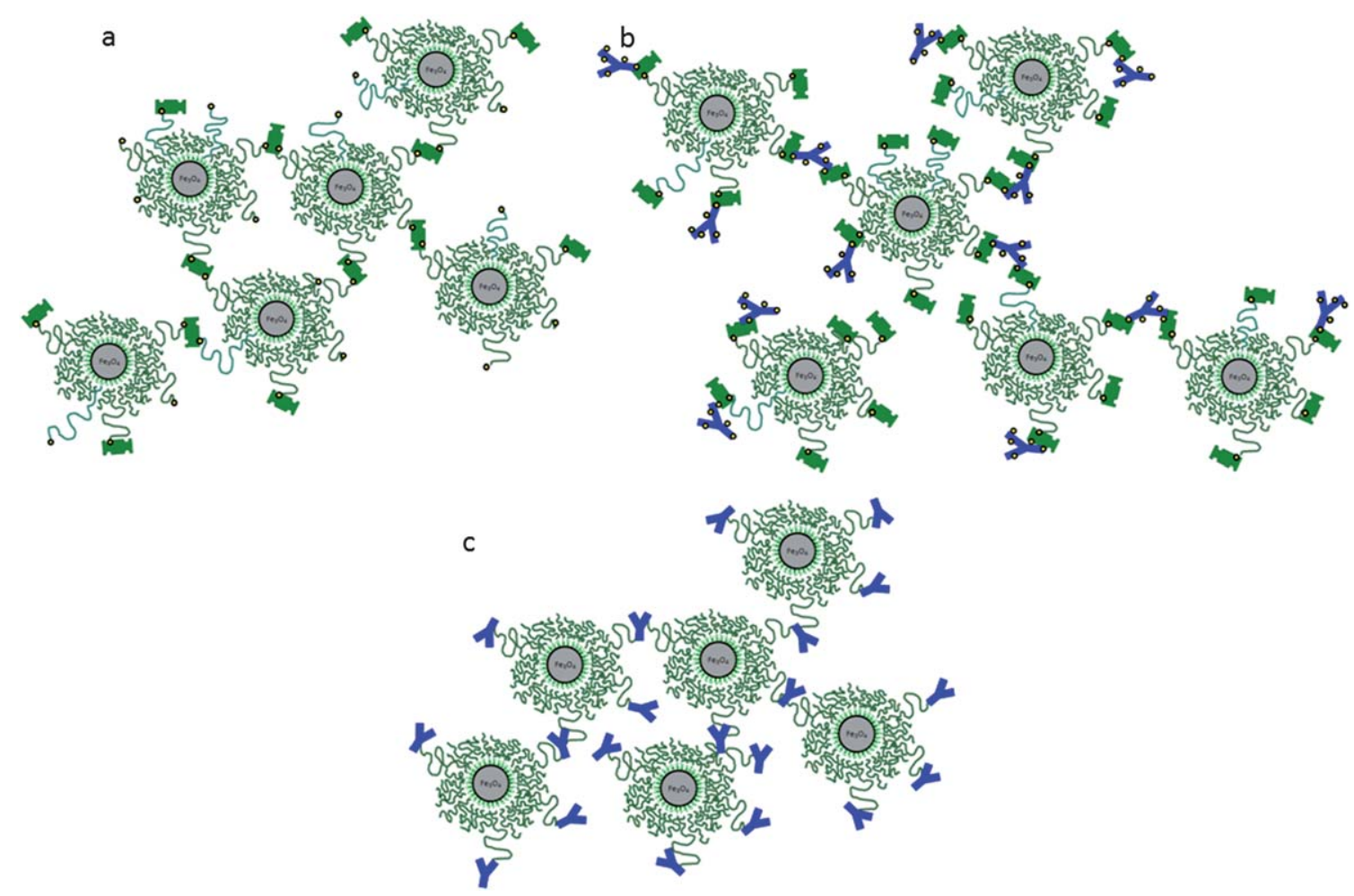

Fig. 9 Schematic of superparamagnetic iron oxide NPs that crosslinked during functionalization. (a) Biotinylated superparamagnetic iron oxide NPs can crosslink during functionalization with avidin derivatives if the molar ratio of avidins : biotinylated iron oxide NPs is too low. (b) Individually stabilized biotinylated superparamagnetic iron oxide NPs that are surrounded by an avidin layer can crosslink during functionalization with biotinylated ligands if the ligands bear more than one biotin per molecule. (c) Superparamagnetic iron oxide NPs can also crosslink when ligands are covalently bound to the NP shell if the ligand contains more than one chemically active group per molecule. Thus, only if ligands contain exactly one chemically active group per molecule through which these ligands can be bound to the NP shell, can crosslinking and thus uncontrolled agglomeration of functionalized superparamagnetic iron oxide NPs be completely avoided.

NP was able to bind to the receptor. ${ }^{181}$ Therefore, the limited number of antibodies that can be immobilized on the NP surface in an orientation that allows ligands to bind to the respective receptor hamper efficient NP targeting. Furthermore, proteins can non-specifically adsorb to antibodies which reduces the circulation time of antibody functionalized NPs. Therefore, ligands that have lower $M_{\mathrm{w}}$ compared to antibodies might be more promising to use as targeting ligands for superparamagnetic iron oxide NPs. ${ }^{181}$

\subsection{Multifunctional iron oxide nanoparticles}

It is often desirable, especially for research purposes, to add multiple labels (imaging modalities), such as fluorophores or radiotracers to iron oxide NP surfaces. A second modality helps us to unequivocally locate such contrast agents and differentiate them from imaging artifacts. The addition of second labels renders superparamagnetic iron oxide NPs multifunctional only if the additional labels are irreversibly bound to their surfaces (Fig. 12). ${ }^{12}$ Table 4 provides an overview of selected multifunctional superparamagnetic iron oxide NPs that were tested in vitro in cell cultures or in vivo.

Combining multiple labels on superparamagnetic iron oxide NPs can lead to undesirable crosstalk. Recently, the combination of plasmonic and magnetic properties within a NP has been demonstrated by spacially separating a plasmonic shell from the iron oxide core through a dielectric polymer layer. ${ }^{197}$ If superparamagnetic iron oxide NPs are functionalized e.g. with near infrared (NIR) fluorescent molecules, fluorescence is hampered by the absorption of light caused by the iron oxide cores. Iron oxide has been reported to quench CY5.5 and FITC if less than two fluorophores were attached to one iron oxide core. ${ }^{198}$ Based on the increased fluorescence observed if these fluorophores were cleaved from the iron oxide NP surface, this quenching was assigned to non-radiative iron oxide-fluorophore interactions. However, for such studies, it is crucial to control the distance of the fluorophores to the iron oxide cores. This could be achieved with low $M_{\mathrm{w}}$ dispersants where fluorophores can be covalently linked to the spacer at a defined distance and minimize the risk that fluorophores directly bind to iron oxide surfaces e.g. through $\mathrm{OH}$ or $\mathrm{SO}^{3-}$ groups.

Purification of superparamagnetic iron oxide NPs stabilized with high $M_{\mathrm{w}}$ dispersants is crucial but difficult. Rigorous purification of such iron oxide NPs risks agglomeration, because the physisorbed dispersants adsorb reversibly at the NP surface. This might be one of the reasons why superparamagnetic iron oxide NPs coated with non-crosslinked dextran or other high $M_{\mathrm{w}}$ dispersants often were not purified with columns (Table 4). However, rigorous purification is 
a

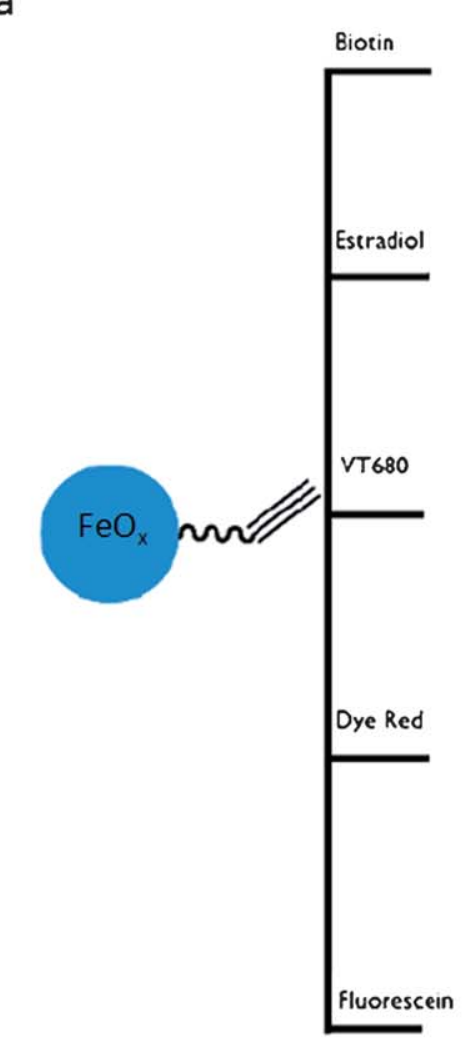

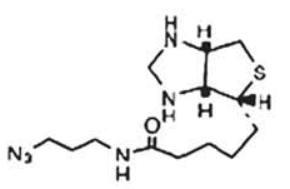<smiles>CNCCN</smiles><smiles>C1CC2CC12</smiles><smiles>C[AsH]</smiles><smiles>C1=CC2CCCC2C1</smiles>
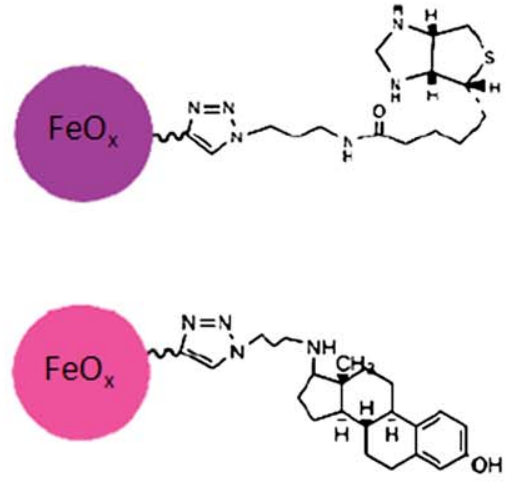

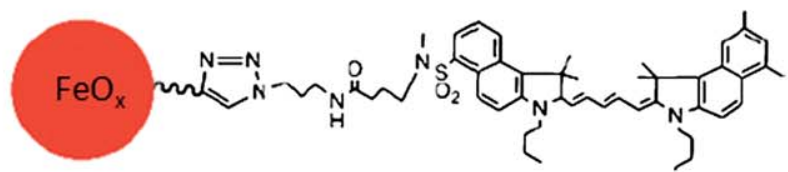<smiles>CN(NCCN)N1CCC(N2CCC([N+](=O)[O-])C2)C1</smiles><smiles></smiles>

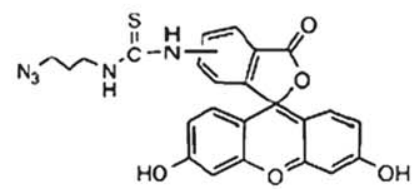

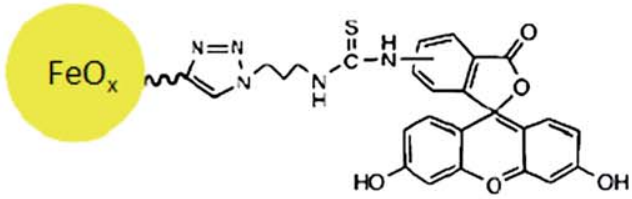

C

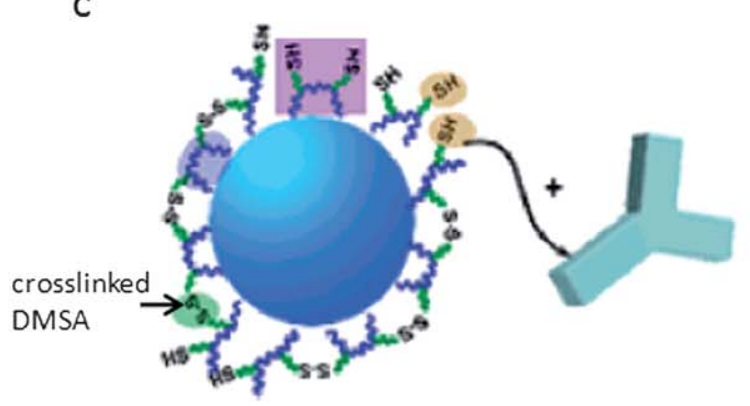

Fig. 10 Functionalization of superparamagnetic iron oxide NPs. Additional functionalities can be imparted on superparamagnetic iron oxide NPs by covalently linking e.g. (a) fluorophores, ${ }^{216}$ (b) radiotracers ${ }^{217}$ (reprinted with permission of Mary Ann Liebert, Inc) and (c) ligands such as antibodies ${ }^{218}$ (Journal of the American Chemical Society by American Chemical Society. Reproduced with permission of AMERICAN CHEMICAL SOCIETY in the format Journal via Copyright Clearance Center.) to the dispersant shell. These additional functionalities can be linked to the dispersant shells with a variety of different chemical reactions. Often used methods are (a) click chemistry or (b and c) thiol, maleimide coupling reactions. Superparamagnetic iron oxide NPs were coated with ( $a$ and $b$ ) dextran and (c) dimercaptosuccinic acid (DMSA) prior to functionalization.

required to eliminate free fluorophores or non-complexed radiotracers. This was exemplified in a study by Jarrett et al. where non-complexed ${ }^{64} \mathrm{Cu}$ could not be removed by centrifugation but required purification of iron oxide NPs by column separation. ${ }^{199}$ Therefore, it is highly beneficial to bind second labels to iron oxide cores through dispersants that adsorb on the NP surface in a well-defined way through suitable, high affinity anchors. If closely packed, these dispersants then allow estimating the distance between optically active tracers and magnetic cores. This is thus key also for controlled assembly of multimodal imaging agents, and ensures that two or more modalities can be co-localized. 

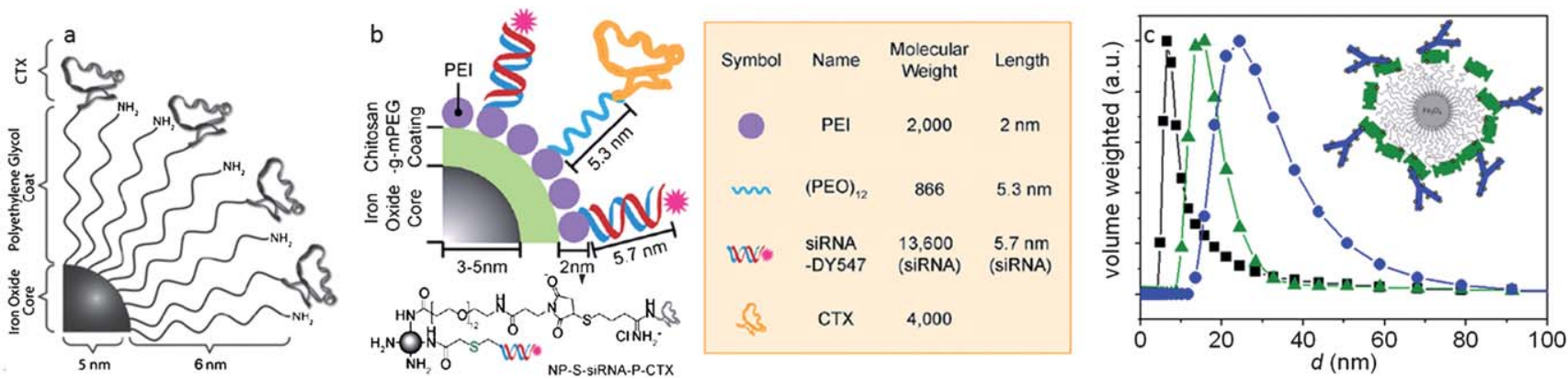

Fig. 11 Effect of functionalization on the size of superparamagnetic iron oxide NPs. (a) The dispersant shell consisting e.g. of amine terminated PEGsilanes $^{219}$ (Copyright Wiley-VCH Verlag GmbH \& Co. KGaA. Reproduced with permission.) can be of comparable size to the iron oxide core. Therefore, the hydrodynamic diameter of sterically stabilized NPs is considerably larger than the core size. (b) Targeting ligands such as chlorotoxin, siRNA and fluorophores can have a similar size to that of the iron oxide cores. Therefore, the hydrodynamic diameter of functionalized NPs can be significantly larger than that of unfunctionalized NPs. ${ }^{220}$ (Biomaterials by Biological Engineering Society. Reproduced with permission of PERGAMON in the format Journal via Copyright Clearance Center.) (c) The increase in hydrodynamic diameter of iron oxide NPs upon coupling ligands to the dispersant shell is experimentally shown on superparamagnetic iron oxide NPs stabilized with PEG (5 kDa)-nitroDOPA that were further functionalized with neutravidin (green) followed by biotinylated antibodies (blue).
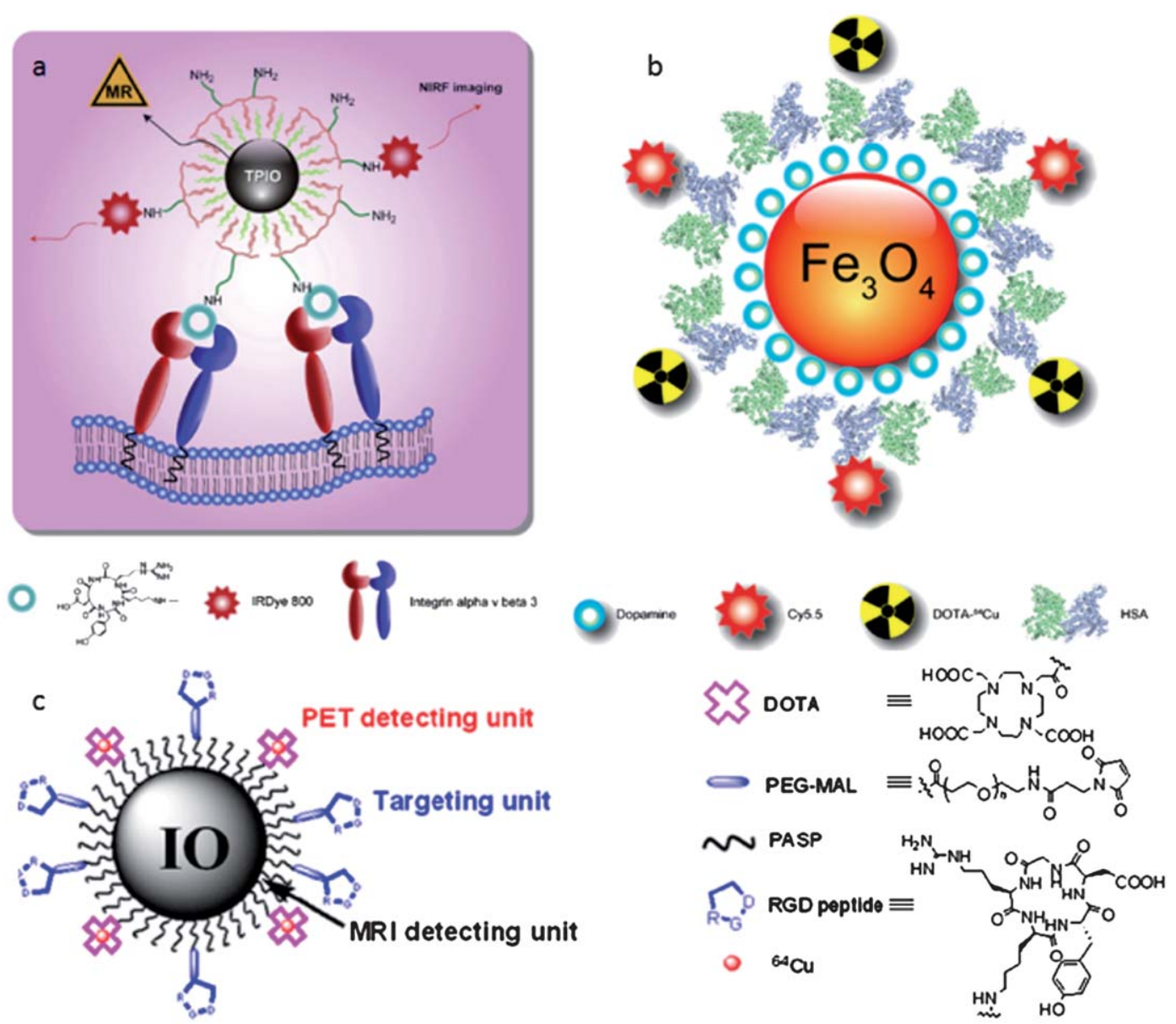

Fig. 12 Assembly of multifunctional contrast agents. Superparamagnetic iron oxide NPs were surface modified with (a) triblock co-polymer consisting of poly(butyl acrylate), poly(ethylene acrylate) and poly(methacrylic acid) yielding TRIPO ${ }^{221}$ (Biomaterials by Biological Engineering Society. Reproduced with permission of PERGAMON in the format Journal via Copyright Clearance Center.), (b) dopamine followed by the adsorption of human serum albumin (HSA) ${ }^{222}$ (Biomaterials by Biological Engineering Society. Reproduced with permission of PERGAMON in the format Journal via Copyright Clearance Center.) and (c) polyaspartic acid (PASP) (reprinted by permission of the Society of Nuclear Medicine from ref. 223). Additional functionalities such as fluorophores, radiotracers and ligands were covalently coupled to the dispersant shell through $\mathrm{NH}_{2}-\mathrm{N}$-hydroxy succinimide (NHS) and maleimide-SH coupling reactions. 


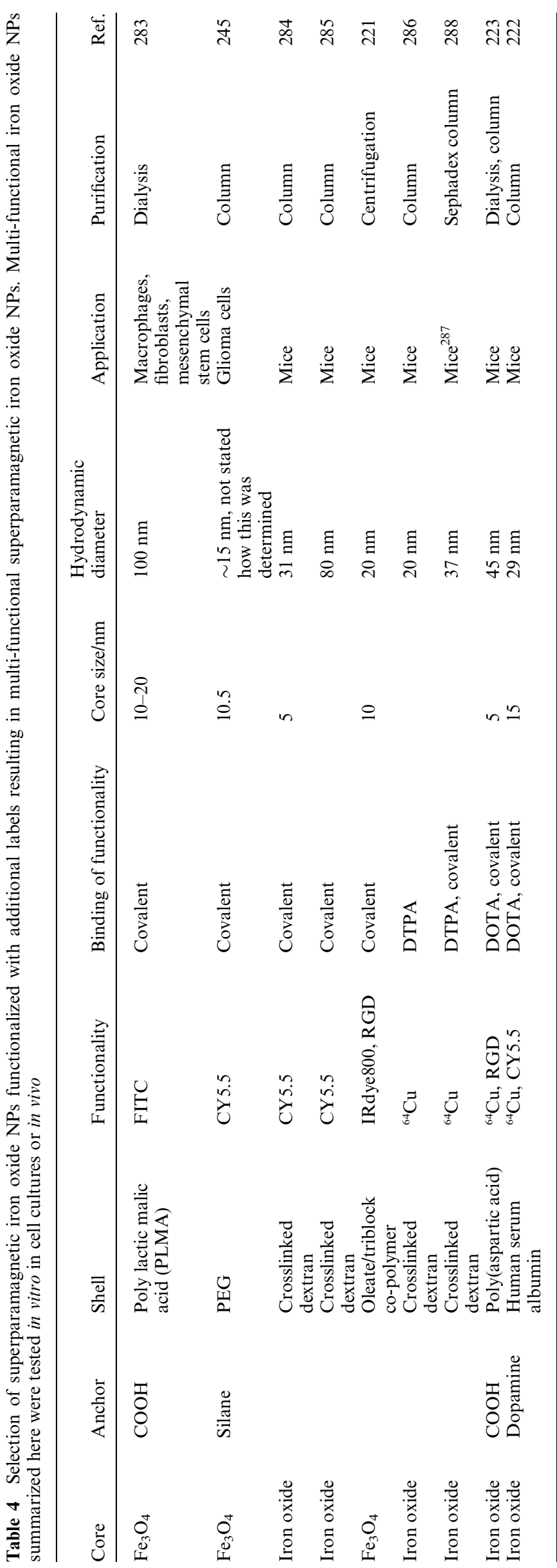

\section{Conclusion}

The increasingly demanding and versatile requirements imposed on superparamagnetic iron oxide NPs intended for biomedical applications require close control over the NP size, structure and surface properties. The key requirement is colloidal stability under physiological conditions which can only be met if iron oxide NPs are sterically stabilized with dispersants that firmly and for practical purposes irreversibly bind to the NP surface. Dispersants consisting of a suitable anchor covalently linked to a spacer have been shown to meet this stringent requirement.

A close control over the assembly of dispersants at the NP surface allows individual tuning of the core size and shell thickness. While the magnetic response of superparamagnetic iron oxide NPs is directly related to the core size, the shell thickness critically affects NP stability and biodistribution. Thus, within the limit of superparamagnetic cores, magnetic response of individually stabilized iron oxide NPs can be maximized by increasing the core size without compromising NP stability. This, however, is only possible if iron oxide NPs are stabilized with optimized dispersants that consist of an irreversibly binding anchor covalently linked to a spacer long enough to provide good steric stability but still small enough to allow high dispersant packing density. If these requirements are fulfilled, individually stabilized superparamagnetic iron oxide NPs can be used as highly stable, well-dispersed NPs for a multitude of biomedical applications. End-grafted and irreversibly bound dispersants further allow for controlled functionalization of individually stabilized NPs. This is achieved by simply adjusting the molar ratio of differently functionalized and unfunctionalized dispersants that are grafted to the iron oxide NP surface.

Thorough characterization of the NP size and surface charge is indispensable for studies where effects of dispersants and ligands are to be studied. Only if NP size and surface charge are similar before and after functionalization can differences in the biodistribution and circulation time be solely assigned to the presence of ligands presented at the NP surfaces. High iron oxide NP concentrations are required for biomedical applications. Therefore, individually stabilized superparamagnetic iron oxide NPs should be functionalized with an optimized low density of ligands and second labels that only possess one chemically active group per molecule. Only then can agglomeration through crosslinking be avoided, and good iron oxide NP stability and close control over the NP size be ensured.

The colloidal and interfacial stability of superparamagnetic iron oxide NPs also has to be considered when evaluating magnetic properties such as saturation magnetization. The saturation magnetization is increased by agglomeration of iron oxide NPs, affecting relaxivity, magnetic separation and SAR, all of relevance for biomedical applications. Furthermore, the anchor chemistry can affect the magnetization through strong interactions with the ions in the surface layer of the magnetic core.

In summary, recent advances in the layer-by-layer assembly of superparamagnetic iron oxide NPs now allow rational design and independent optimization of the many different required properties of iron oxide NPs. The key aspect is a well-defined dispersant shell which consists of spacers that are sufficiently strongly anchored to the iron oxide NP at high packing densities. 
If this requirement is fulfilled, additional imaging modalities can be added to the NP surface in a controlled way by co-adsorbing differently end-functionalized dispersants. This modular approach greatly enhances the versatility of applying NP platforms to the various biomedical applications they are being designed for, but requires thorough interdisciplinary characterization that is not always performed for every material study. The critical evaluation in this review of the current state-of-theart in the field, however, demonstrates that there is great potential in the near future for further breakthrough developments in NP design for biomedical and other applications.

\section{References}

1 M. Lewin, N. Carlesso, C. H. Tung, X. W. Tang, D. Cory, D. T. Scadden and R. Weissleder, Nat. Biotechnol., 2000, 18(4), 410-414.

2 M. J. Pittet, F. K. Swirski, F. Reynolds, L. Josephson and R. Weissleder, Nat. Protoc., 2006, 1, 73-79.

3 D. S. Wang, J. B. He, N. Rosenzweig and Z. Rosenzweig, Nano Lett., 2004, 4(3), 409-413.

4 A. Halbreich, J. Roger, J. N. Pons, D. Geldwerth, M. F. Da Silva, M. Roudier and J. C. Bacri, Biochimie, 1998, 80(5-6), 379-390.

5 Q. A. Pankhurst, N. K. T. Thanh, S. K. Jones and J. Dobson, J. Phys. D: Appl. Phys., 2009, 42(22), 224001.

6 M. Namdeo, S. Saxena, R. Tankhiwale, M. Bajpai, Y. M. Mohan and S. K. Bajpai, J. Nanosci. Nanotechnol., 2008, 8(7), 3247-3271.

7 R. Weissleder, P. F. Hahn, D. D. Stark, E. Rummeny, S. Saini, J. Wittenberg and J. T. Ferrucci, Am. J. Roentgenol., 1987, 149(4), 723-726.

8 R. Weissleder, G. Elizondo, J. Wittenberg, C. A. Rabito, H. H. Bengele and L. Josephson, Radiology, 1990, 175(2), 489-493.

9 J. R. McCarthy and R. Weissleder, Adv. Drug Delivery Rev., 2008, 60 (11), 1241-1251.

10 N. Lewinski, V. Colvin and R. Drezek, Small, 2008, 4(1), 26-49.

11 K. M. Krishnan, IEEE Trans. Magn., 2010, 46(7), 2523-2558.

12 A. Louie, Chem. Rev., 2010, 110(5), 3146-3195.

13 Y. X. J. Wang, S. M. Hussain and G. P. Krestin, Eur. Radiol., 2001, 11(11), 2319-2331.

14 A. Hutten, D. Sudfeld, I. Ennen, G. Reiss, W. Hachmann, U. Heinzmann, K. Wojczykowski, P. Jutzi, W. Saikaly and G. Thomas, J. Biotechnol., 2004, 112(1-2), 47-63.

15 J. D. G. Duran, J. L. Arias, V. Gallardo and A. V. Delgado, J. Pharm. Sci., 2008, 97(8), 2948-2983.

16 C. W. Jung and P. Jacobs, Magn. Reson. Imaging, 1995, 13(5), 661674.

17 M. M. Lin, D. K. Kim, A. J. El Haj and J. Dobson, IEEE Trans. NanoBiosci., 2008, 7(4), 298-305.

18 F. Cengelli, D. Maysinger, F. Tschudi-Monnet, X. Montet, C. Corot, A. Petri-Fink, H. Hofmann and L. Juillerat-Jeanneret, $J$. Pharmacol. Exp. Ther., 2006, 318(1), 108-116.

19 H. Pardoe, W. Chua-anusorn, T. G. St Pierre and J. Dobson, $J$. Magn. Magn. Mater., 2001, 225(1-2), 41-46.

20 M. C. Bautista, O. Bomati-Miguel, X. Zhao, M. P. Morales, T. Gonzalez-Carreno, R. P. de Alejo, J. Ruiz-Cabello and S. Veintemillas-Verdaguer, Nanotechnology, 2004, 15(4), S154-S159.

21 S. K. Basiruddin, A. Saha, N. Pradhan and N. R. Jana, J. Phys. Chem. C, 2010, 114(25), 11009-11017.

22 E. Amstad, S. Zurcher, A. Mashaghi, J. Y. Wong, M. Textor and E. Reimhult, Small, 2009, 5(11), 1334-1342.

23 E. Amstad, T. Gillich, I. Bilecka, M. Textor and E. Reimhult, Nano Lett., 2009, 9(12), 4042-4048.

24 A. H. Lu, E. L. Salabas and F. Schuth, Angew. Chem., Int. Ed., 2007, 46(8), 1222-1244.

25 Y. W. Jun, J. H. Lee and J. Cheon, Angew. Chem., Int. Ed., 2008, 47 (28), 5122-5135.

26 S. H. Sun, H. Zeng, D. B. Robinson, S. Raoux, P. M. Rice, S. X. Wang and G. X. Li, J. Am. Chem. Soc., 2004, 126(1), 273-279.

27 A. F. Thunemann, S. Rolf, P. Knappe and S. Weidner, Anal. Chem., 2009, 81(1), 296-301.
28 M. Bonini, A. Wiedemann and P. Baglioni, Mater. Sci. Eng., C, 2006, 26(5-7), 745-750.

29 K. Butter, A. Hoell, A. Wiedenmann, A. V. Petukhov and G. J. Vroege, J. Appl. Crystallogr., 2004, 37, 847-856.

30 P. Degen, A. Shukla, U. Boetcher and H. Rehage, Colloid Polym. Sci., 2008, 286(2), 159-168.

31 R. J. H. Cowles, Pet. Sci. Technol., 1999, 17(3-4), 429-442.

32 P. Roonasi and A. Holmgren, Appl. Surf. Sci., 2009, 255(11), 58915895.

33 S. Chen, Y. Li, C. Guo, J. Wang, J. H. Ma, X. F. Liang, L. R. Yang and H. Z. Liu, Langmuir, 2007, 23, 12669-12676.

34 M. Zackrisson, A. Stradner, P. Schurtenberger and J. Bergenholtz, Langmuir, 2005, 21(23), 10835-10845.

35 N. Dingenouts, S. Seelenmeyer, I. Deike, S. Rosenfeldt, M. Ballau, P. Lindner and T. Narayanan, Phys. Chem. Chem. Phys., 2001, 3 (7), 1169-1174.

36 T. Gelbrich, M. Feyen and A. M. Schmidt, Macromolecules, 2006, 39 (9), 3469-3472.

37 P. Zakharov, S. Bhat, P. Schurtenberger and F. Scheffold, Appl. Opt., 2006, 45(8), 1756-1764.

38 F. Scheffold and T. G. Mason, J. Phys.: Condens. Matter, 2009, 21 (33), 332102.

39 Z. P. Xiao, K. M. Yang, H. Liang and J. Lu, J. Polym. Sci., Part A: Polym. Chem., 2010, 48(3), 542-550.

40 K. Somaskandan, T. Veres, M. Niewczas and B. Simard, New J. Chem., 2008, 32(2), 201-209.

41 E. Amstad, L. W. E. Starmans, M. A. Visbal, L. Isa, C. Rinaldi, M. Textor and E. Reimhult, in preparation.

42 Q. A. Zhang, M. S. Thompson, A. Y. Carmichael-Baranauskas, B. L. Caba, M. A. Zalich, Y. N. Lin, O. T. Mefford, R. M. Davis and J. S. Riffle, Langmuir, 2007, 23(13), 6927-6936.

43 O. T. Mefford, M. L. Vadala, J. D. Goff, M. R. J. Carroll, R. MejiaAriza, B. L. Caba, T. G. S. Pierre, R. C. Woodward, R. M. Davis and J. S. Riffle, Langmuir, 2008, 24(9), 5060-5069.

44 M. A. Bevan, S. N. Petris and D. Y. C. Chan, Langmuir, 2002, 18 (21), 7845-7852.

45 N. T. K. Thanh and L. A. W. Green, Nano Today, 2010, 5(3), 213230.

46 A. Verma and F. Stellacci, Small, 2010, 6(1), 12-21.

47 L. F. Gamarra, E. Amaro, S. Alves, D. Soga, W. M. Pontuschka, J. B. Mamani, S. M. Carneiro, G. E. S. Brito and A. M. F. Neto, J. Nanosci. Nanotechnol., 2010, 10(7), 4145-4153.

48 H. L. Ma, X. T. Qi, Y. Maitani and T. Nagai, Int. J. Pharm., 2007, 333(1-2), 177-186.

49 J. H. Park, K. H. Im, S. H. Lee, D. H. Kim, D. Y. Lee, Y. K. Lee, K. M. Kim and K. N. Kim, J. Magn. Magn. Mater., 2005, 293(1), 328-333.

50 M. Mahmoudi, A. Simchi, A. S. Milani and P. Stroeve, J. Colloid Interface Sci., 2009, 336(2), 510-518.

51 A. Chastellain, A. Petri and H. Hofmann, J. Colloid Interface Sci., 2004, 278(2), 353-360.

52 B. Schopf, T. Neuberger, K. Schulze, A. Petri, M. Chastellain, M. Hofmann, H. Hofmann and B. von Rechenberg, J. Magn. Magn. Mater., 2005, 293(1), 411-418.

53 S. Santra, C. Kaittanis, J. Grimm and J. M. Perez, Small, 2009, 5 (16), 1862-1868.

54 A. F. Thunemann, D. Schutt, L. Kaufner, U. Pison and H. Mohwald, Langmuir, 2006, 22(5), 2351-2357.

55 V. I. Shubayev, T. R. Pisanic and S. H. Jin, Adv. Drug Delivery Rev., $2009,61(6), 467-477$.

56 V. C. F. Mosqueira, P. Legrand, J. L. Morgat, M. Vert, E. Mysiakine, R. Gref, J. P. Devissaguet and G. Barratt, Pharm. Res., 2001, 18(10), 1411-1419.

57 L. Josephson, C. H. Tung, A. Moore and R. Weissleder, Bioconjugate Chem., 1999, 10(2), 186-191.

58 S. Laurent, D. Forge, M. Port, A. Roch, C. Robic, L. V. Elst and R. N. Muller, Chem. Rev., 2008, 108(6), 2064-2110.

59 C. Corot, P. Robert, J. M. Idee and M. Port, Adv. Drug Delivery Rev., 2006, 58(14), 1471-1504.

60 B. Y. Du, A. X. Mei, P. J. Tao, B. Zhao, Z. Cao, J. J. Nie, J. T. Xu and Z. Q. Fan, J. Phys. Chem. C, 2009, 113(23), 1009010096

61 S. X. Wang, Y. Zhou, W. Guan and B. J. Ding, Appl. Surf. Sci., 2008, 254(16), 5170-5174.

62 B. Zhao and W. J. Brittain, Prog. Polym. Sci., 2000, 25(5), 677-710. 
63 K. H. Bae, Y. B. Kim, Y. Lee, J. Hwang, H. Park and T. G. Park, Bioconjugate Chem., 2010, 21(3), 505-512.

64 K. Nagase, J. Kobayashi and T. Okano, J. R. Soc. Interface, 2009, S293-S309.

65 W. Knoll and R. C. Advincula, Functional Polymer Films, WileyVCH, 2011, vol. 2.

66 J. Xie, C. J. Xu, Z. C. Xu, Y. L. Hou, K. L. Young, S. X. Wang, N. Pourmond and S. H. Sun, Chem. Mater., 2006, 18(23), 54015403.

67 J. Xie, K. Chen, H.-Y. Lee, C. Xu, A. R. Hsu, S. Peng, X. Chen and $\mathrm{S}$. Sun, Ultrasmall c(RGDyK)-coated $\mathrm{Fe}_{3} \mathrm{O}_{4}$ nanoparticles and their specific targeting to integrin alpha(v)beta3-rich tumor cells, J. Am. Chem. Soc., 2008, 130, 7542-7543.

68 C. J. Xu, K. M. Xu, H. W. Gu, R. K. Zheng, H. Liu, X. X. Zhang, Z. H. Guo and B. Xu, J. Am. Chem. Soc., 2004, 126(32), 99389939.

69 H. W. Gu, Z. M. Yang, J. H. Gao, C. K. Chang and B. Xu, J. Am. Chem. Soc., 2005, 127(1), 34-35.

70 E. Amstad, L. Isa and E. Reimhult, Chimia, 2011, 64(11), 826.

71 L. Isa, E. Amstad, M. Textor and E. Reimhult, Chimia, 2010, 64(3), 145-149.

72 S. Yu and G. M. Chow, J. Mater. Chem., 2004, 14(18), 2781-2786.

73 M. A. White, J. A. Johnson, J. T. Koberstein and N. J. Turro, J. Am. Chem. Soc., 2006, 128(35), 11356-11357.

74 H. T. Song, J. S. Choi, Y. M. Huh, S. Kim, Y. W. Jun, J. S. Suh and J. Cheon, J. Am. Chem. Soc., 2005, 127(28), 9992-9993.

75 B. Basly, D. Felder-Flesch, P. Perriat, C. Billotey, J. Taleb, G. Pourroy and S. Begin-Colin, Chem. Commun., 2010, 46(6), 985987.

76 Y. Lalatonne, C. Paris, J. M. Serfaty, P. Weinmann, M. Lecouvey and L. Motte, Chem. Commun., 2008, (22), 2553-2555.

77 Y. Zhou, S. X. Wang, B. J. Ding and Z. M. Yang, Chem. Eng. J., 2008, 138(1-3), 578-585.

78 D. Forge, S. Laurent, Y. Gossuin, A. Roch, L. V. Elst and R. N. Muller, J. Magn. Magn. Mater., 2011, 323(5), 410415.

79 C. R. Sun, K. Du, C. Fang, N. Bhattarai, O. Veiseh, F. Kievit, Z. Stephen, D. H. Lee, R. G. Ellenbogen, B. Ratner and M. Q. Zhang, ACS Nano, 2010, 4(4), 2402-2410.

80 O. Veiseh, J. W. Gunn, F. M. Kievit, C. Sun, C. Fang, J. S. H. Lee and M. Q. Zhang, Small, 2009, 5(2), 256-264.

81 E. K. U. Larsen, T. Nielsen, T. Wittenborn, H. Birkedal, T. VorupJensen, M. H. Jakobsen, L. Ostergaard, M. R. Horsman, F. Besenbacher, K. A. Howard and J. Kjems, ACS Nano, 2009, 3 (7), 1947-1951.

82 J. L. Dalsin, L. J. Lin, S. Tosatti, J. Voros, M. Textor and P. B. Messersmith, Langmuir, 2005, 21(2), 640-646.

83 M. K. Corbierre, N. S. Cameron and R. B. Lennox, Langmuir, 2004, 20(7), 2867-2873.

84 M. Kim, Y. F. Chen, Y. C. Liu and X. G. Peng, Adv. Mater., 2005, 17(11), 1429.

85 Z. P. Chen, Y. Zhang, K. Xu, R. Z. Xu, J. W. Liu and N. Gu, J. Nanosci. Nanotechnol., 2008, 8(12), 6260-6265.

86 C. Haensch, M. Chiper, C. Ulbricht, A. Winter, S. Hoeppener and U. S. Schubert, Langmuir, 2008, 24(22), 12981-12985.

87 J. H. Waite and M. L. Tanzer, Science, 1981, 212(4498), 1038-1040.

88 M. W. Lynch, M. Valentine and D. N. Hendrickson, J. Am. Chem. Soc., 1982, 104(25), 6982-6989.

89 R. H. Heistand, A. L. Roe and L. Que, Inorg. Chem., 1982, 21(2), 676-681.

90 A. S. Attia, S. Bhattacharya and C. G. Pierpont, Inorg. Chem., 1995, 34(17), 4427-4433.

91 V. A. Grillo, G. R. Hanson, D. M. Wang, T. W. Hambley, L. R. Gahan, K. S. Murray, B. Moubaraki and C. J. Hawkins, Inorg. Chem., 1996, 35(12), 3568-3576.

92 J. J. Girerd, M. L. Boillot, G. Blain and E. Riviere, Inorg. Chim. Acta, 2008, 361(14-15), 4012-4016.

93 J. T. Weisser, M. J. Nilges, M. J. Sever and J. J. Wilker, Inorg. Chem., 2006, 45(19), 7736-7747.

94 M. J. Sever, J. T. Weisser, J. Monahan, S. Srinivasan and J. J. Wilker, Angew. Chem., Int. Ed., 2004, 43(4), 448-450.

95 R. B. Lauffer, R. H. Heistand and L. Que, Inorg. Chem., 1983, 22(1), $50-55$.

96 A. P. S. Citadini, A. P. A. Pinto, A. P. U. Araujo, O. R. Nascimento and A. J. Costa, Biophys. J., 2005, 88(5), 3502-3508.
97 P. C. A. Bruijnincx, M. Lutz, A. L. Spek, W. R. Hagen, G. van Koten and R. Gebbink, Inorg. Chem., 2007, 46, 8391-8402.

98 B. Kalyanaraman, C. C. Felix and R. C. Sealy, Environ. Health Perspect., 1985, 64, 185-198.

99 D. D. Cox and L. Que, J. Am. Chem. Soc., 1988, 110(24), 8085-8092.

100 J. P. Emerson, E. G. Kovaleva, E. R. Farquhar, J. D. Lipscomb and L. Que, Proc. Natl. Acad. Sci. U. S. A., 2008, 105(21), 7347-7352.

101 M. D. Shultz, J. U. Reveles, S. N. Khanna and E. E. Carpenter, J. Am. Chem. Soc., 2007, 129(9), 2482-2487.

102 A. S. Goldmann, C. Schodel, A. Walther, J. Y. Yuan, K. Loos and A. H. E. Muller, Macromol. Rapid Commun., 2010, 31(18), 16081615.

103 U. ElAyaan, E. Herlinger, R. F. Jameson and W. Linert, J. Chem. Soc., Dalton Trans., 1997, (16), 2813-2818.

104 J. R. Galpin, L. G. M. Tielens, G. A. Veldink, J. F. G. Vliegenthart and J. Boldingh, FEBS Lett., 1976, 69(1), 179-182.

105 T. Kawabata, V. Schepkin, N. Haramaki, R. S. Phadke and L. Packer, Biochem. Pharmacol., 1996, 51(11), 1569-1577.

106 G. Crisponi and M. Remelli, Coord. Chem. Rev., 2008, 252(10-11), $1225-1240$.

107 V. M. Nurchi, T. Pivetta, J. I. Lachowicz and G. Crisponi, J. Inorg. Biochem., 2009, 103(2), 227-236.

108 E. Amstad, A. U. Gehring, H. Fischer, V. V. Nagaiyanallur, G. Haehner, M. Textor and E. Reimhult, J. Phys. Chem. C, 2010, 115(3), 683-691.

109 E. Amstad, H. Fischer, A. U. Gehring, M. Textor and E. Reimhult, Chem.-Eur. J., 2011, DOI: 10.1002/chem.201003504.

110 D. Vasudevan and A. T. Stone, J. Colloid Interface Sci., 1998, 202(1), $1-19$.

111 D. Vasudevan and A. T. Stone, Environ. Sci. Technol., 1996, 30(5), $1604-1613$

112 S. T. Martin, J. M. Kesselman, D. S. Park, N. S. Lewis and M. R. Hoffmann, Environ. Sci. Technol., 1996, 30(8), 2535-2542.

113 B. Malisova, S. Tosatti, M. Textor, K. Gademann and S. Zurcher, Langmuir, 2010, 26(6), 4018-4026.

114 G. Fritz, V. Schadler, N. Willenbacher and N. J. Wagner, Langmuir, 2002, 18(16), 6381-6390.

115 A. P. Gast, Langmuir, 1996, 12(17), 4060-4067.

116 T. A. Witten and P. A. Pincus, Macromolecules, 1986, 19(10), 25092513.

117 B. Vincent, J. Edwards, S. Emmett and A. Jones, Colloids Surf., 1986, 18(2-4), 261-281.

118 P. G. Degennes, Macromolecules, 1980, 13(5), 1069-1075.

119 S. T. Milner, T. A. Witten and M. E. Cates, Macromolecules, 1988, 21(8), 2610-2619.

120 E. B. Zhulina, O. V. Borisov and V. A. Priamitsyn, J. Colloid Interface Sci., 1990, 137(2), 495-511.

121 A. F. Miller, R. W. Richards and J. R. P. Webster, Macromolecules, 2001, 34(23), 8361-8369.

122 S. T. Milner, T. A. Witten and M. E. Cates, Europhys. Lett., 1988, 5 (5), 413-418.

123 D. F. K. Shim and M. E. Cates, J. Phys., 1989, 50(24), 3535-3551.

124 S. Alexander, J. Phys., 1977, 38(8), 977-981.

125 T. M. Birshtein and E. B. Zhulina, Polymer, 1984, 25(10), 14531461.

126 N. Dan and M. Tirrell, Macromolecules, 1992, 25(11), 2890-2895.

127 R. Toral and A. Chakrabarti, Phys. Rev. E: Stat. Phys., Plasmas, Fluids, Relat. Interdiscip. Top., 1993, 47(6), 4240-4246.

128 E. K. Lin and A. P. Gast, Macromolecules, 1996, 29(1), 390-397.

129 R. C. Ball, J. F. Marko, S. T. Milner and T. A. Witten, Macromolecules, 1991, 24(3), 693-703.

$130 \mathrm{H}$. Li and T. A. Witten, Macromolecules, 1994, 27(2), 449-457.

131 J. I. Martin and Z. G. Wang, J. Phys. Chem., 1995, 99(9), 2833-2844.

132 K. A. Cogan, A. P. Gast and M. Capel, Macromolecules, 1991, 24 (24), 6512-6520.

133 J. S. Pedersen and C. Svaneborg, Curr. Opin. Colloid Interface Sci., 2002, 7(3-4), 158-166.

134 J. S. Pedersen and M. C. Gerstenberg, Colloids Surf., A, 2003, 213(23), 175-187.

135 T. Riley, C. R. Heald, S. Stolnik, M. C. Garnett, L. Illum, S. S. Davis, S. M. King, R. K. Heenan, S. C. Purkiss, R. J. Barlow, P. R. Gellert and C. Washington, Langmuir, 2003, 19(20), 8428-8435.

136 D. E. Owens and N. A. Peppas, Int. J. Pharm., 2006, 307(1), 93102. 
137 S. I. Jeon, J. H. Lee, J. D. Andrade and P. G. Degennes, J. Colloid Interface Sci., 1991, 142(1), 149-158.

138 R. Bhat and S. N. Timasheff, Protein Sci., 1992, 1(9), 11331143.

139 K. Feldman, G. Hahner, N. D. Spencer, P. Harder and M. Grunze, J. Am. Chem. Soc., 1999, 121(43), 10134-10141.

140 R. L. C. Wang, H. J. Kreuzer and M. Grunze, J. Phys. Chem. B, 1997, 101(47), 9767-9773.

141 A. Roosjen, J. de Vries, H. C. van der Mei, W. Norde and H. J. Busscher, J. Biomed. Mater. Res., Part B, 2005, 73(2), 347-354.

142 M. C. Shen, L. Martinson, M. S. Wagner, D. G. Castner, B. D. Ratner and T. A. Horbett, J. Biomater. Sci., Polym. Ed., 2002, 13(4), 367-390.

143 R. Konradi, B. Pidhatika, A. Muhlebach and M. Textort, Langmuir, 2008, 24(3), 613-616.

144 S. Pasche, S. M. De Paul, J. Voros, N. D. Spencer and M. Textor, Langmuir, 2003, 19(22), 9216-9225.

145 R. Michel, S. Pasche, M. Textor and D. G. Castner, Langmuir, 2005, 21(26), 12327-12332.

146 I. Szleifer, Biophys. J., 1997, 72(2), 595-612.

147 A. S. Zahr, C. A. Davis and M. V. Pishko, Langmuir, 2006, 22(19), $8178-8185$.

148 A. Gessner, B. R. Paulke, R. H. Muller and T. M. Goppert, Pharmazie, 2006, 61(4), 293-297.

149 A. K. Kenworthy, K. Hristova, D. Needham and T. J. McIntosh, Biophys. J., 1995, 68(5), 1921-1936.

150 M. Vittaz, D. Bazile, G. Spenlehauer, T. Verrecchia, M. Veillard, F. Puisieux and D. Labarre, Biomaterials, 1996, 17(16), 15751581.

151 G. Storm, S. O. Belliot, T. Daemen and D. D. Lasic, Adv. Drug Delivery Rev., 1995, 17(1), 31-48.

152 A. L. Klibanov, K. Maruyama, V. P. Torchilin and L. Huang, FEBS Lett., 1990, 268(1), 235-237.

153 A. Mori, A. L. Klibanov, V. P. Torchilin and L. Huang, FEBS Lett., 1991, 284(2), 263-266.

154 P. Decuzzi, R. Pasqualini, W. Arap and M. Ferrari, Pharm. Res., 2009, 26(1), 235-243.

155 J. K. Gbadamosi, A. C. Hunter and S. M. Moghimi, FEBS Lett., 2002, 532(3), 338-344.

156 M. A. Dobrovolskaia and S. E. McNeil, Nat. Nanotechnol., 2007, 2 (8), 469-478.

157 L. X. Tiefenauer, A. Tschirky, G. Kuhne and R. Y. Andres, Magn. Reson. Imaging, 1996, 14(4), 391-402.

158 G. R. Harper, M. C. Davies, S. S. Davis, T. F. Tadros, D. C. Taylor, M. P. Irving and J. A. Waters, Biomaterials, 1991, 12(7), 695-704.

159 L. M. Lacava, V. A. P. Garcia, S. Kuckelhaus, R. B. Azevedo, N. Sadeghiani, N. Buske, P. C. Morais and Z. G. M. Lacava, J. Magn. Magn. Mater., 2004, 272, 2434-2435.

160 A. Berkowit, W. J. Schuele and P. J. Flanders, J. Appl. Phys., 1968, 39(2P2), 1261.

161 P. Dutta, S. Pai, M. S. Seehra, N. Shah and G. P. Huffman, J. Appl. Phys., 2009, 105(7), 073104

162 K. L. Krycka, R. A. Booth, C. R. Hogg, Y. Ijiri, J. A. Borchers, W. C. Chen, S. M. Watson, M. Laver, T. R. Gentile, L. R. Dedon, S. Harris, J. J. Rhyne and S. A. Majetich, Phys. Rev. Lett., 2010, 104(20), 207203.

163 J. Vidal-Vidal, J. Rivas and M. A. Lopez-Quintela, Colloids Surf., A, 2006, 288(1-3), 44-51.

164 Y. W. Jun, Y. M. Huh, J. S. Choi, J. H. Lee, H. T. Song, S. Kim, S. Yoon, K. S. Kim, J. S. Shin, J. S. Suh and J. Cheon, J. Am. Chem. Soc., 2005, 127(16), 5732-5733.

165 J. Cheon and J. H. Lee, Acc. Chem. Res., 2008, 41(12), 1630-1640.

166 J. F. Berret, N. Schonbeck, F. Gazeau, D. El Kharrat, O. Sandre, A. Vacher and M. Airiau, J. Am. Chem. Soc., 2006, 128(5), 17551761.

167 S. B. Seo, J. Yang, T. I. Lee, C. H. Chung, Y. J. Song, J. S. Suh, H. G. Yoon, Y. M. Huh and S. Haam, J. Colloid Interface Sci., 2008, 319(2), 429-434.

168 L. Josephson, J. M. Perez and R. Weissleder, Angew. Chem., Int. Ed., 2001, 40(17), 3204.

169 K. A. Brown, C. C. Vassiliou, D. Issadore, J. Berezovsky, M. J. Cima and R. M. Westervelt, J. Magn. Magn. Mater., 2010, 322(20), 3122-3126.

170 Y. Matsumoto and A. Jasanoff, Magn. Reson. Imaging, 2008, 26(7), 994-998.
171 L. E. W. LaConte, N. Nitin, O. Zurkiya, D. Caruntu, C. J. O'Connor, X. P. Hu and G. Bao, J. Magn. Reson. Imaging, 2007, 26(6), 1634-1641.

172 H. W. Duan, M. Kuang, X. X. Wang, Y. A. Wang, H. Mao and S. M. Nie, J. Phys. Chem. C, 2008, 112(22), 8127-8131.

173 R. E. Rosensweig, J. Magn. Magn. Mater., 2002, 252(1-3), 370-374.

174 K. Buscher, C. A. Helm, C. Gross, G. Glockl, E. Romanus and W. Weitschies, Langmuir, 2004, 20(6), 2435-2444.

175 S. A. Rovers, C. Dietz, L. A. M. van der Poel, R. Hoogenboom, M. F. Kemmere and J. T. F. Keurentjes, J. Phys. Chem. C, 2010, 114(18), 8144-8149.

176 D. E. Sosnovik, M. Nahrendorf and R. Weissleder, Basic Res. Cardiol., 2008, 103(2), 122-130.

177 H. Z. Jin and K. A. Kang, Oxygen Transport to Tissue Xxviii, 2007, vol. 599 , pp. $45-52$.

178 A. H. Latham and M. E. Williams, Acc. Chem. Res., 2008, 41(3), $411-420$.

179 A. Tsourkas, V. R. Shinde-Patil, K. A. Kelly, P. Patel, A. Wolley, J. R. Allport and R. Weissleder, Bioconjugate Chem., 2005, 16(3), $576-581$.

180 K. A. Kelly, J. R. Allport, A. Tsourkas, V. R. Shinde-Patil, L. Josephson and R. Weissleder, Circ. Res., 2005, 96(3), 327-336.

181 X. Montet, M. Funovics, K. Montet-Abou, R. Weissleder and L. Josephson, J. Med. Chem., 2006, 49(20), 6087-6093.

182 A. L. Martin, J. L. Hickey, A. L. Ablack, J. D. Lewis, L. G. Luyt and E. R. Gillies, J. Nanopart. Res., 2010, 12(5), 1599-1608.

183 M. V. Yigit, D. Mazumdar, H. K. Kim, J. H. Lee, B. Dintsov and Y. Lu, ChemBioChem, 2007, 8, 1675-1678.

184 J. I. Cutler, D. Zheng, X. Y. Xu, D. A. Giljohann and C. A. Mirkin, Nano Lett., 2010, 10(4), 1477-1480.

185 O. Veiseh, F. M. Kievit, C. Fang, N. Mu, S. Jana, M. C. Leung, H. Mok, R. G. Ellenbogen, J. O. Park and M. Zhang, Biomaterials, 2010, 31(31), 8032-8042.

186 E. C. Cho, C. Glaus, J. Y. Chen, M. J. Welch and Y. N. Xia, Trends Mol. Med., 2010, 16(12), 561-573.

187 M. E. Gindy and R. K. Prud'homme, Expert Opin. Drug Delivery, 2009, 6(8), 865-878.

188 C. Sun, J. S. H. Lee and M. Q. Zhang, Adv. Drug Delivery Rev., 2008, 60(11), 1252-1265.

189 N. M. Green, Methods Enzymol., 1990, 184, 51-67.

190 S. C. Meyer, T. Gaj and I. Ghosh, Chem. Biol. Drug Des., 2006, 68 (1), 3-10.

191 R. Narain, M. Gonzales, A. S. Hoffman, P. S. Stayton and K. M. Krishnan, Langmuir, 2007, 23(11), 6299-6304.

192 J. J. Lai, J. M. Hoffman, M. Ebara, A. S. Hoffman, C. Estournes, A. Wattiaux and P. S. Stayton, Langmuir, 2007, 23(13), 7385-7391.

193 P. Vermette, S. Taylor, D. Dunstan and L. Meagher, Langmuir, 2002, 18(2), 505-511.

194 E. T. Kisak, M. T. Kennedy, D. Trommeshauser and J. A. Zasadzinski, Langmuir, 2000, 16(6), 2825-2831.

195 J. Huang, L. H. Bu, J. Xie, K. Chen, Z. Cheng, X. G. Li and X. Y. Chen, ACS Nano, 2011, 4(12), 7151-7160.

196 M. K. Yu, J. Park, Y. Y. Jeong, W. K. Moon and S. Jon, Nanotechnology, 2010, 21(41), 415102.

197 Y. Jin, C. Jia, S.-W. Huang, M. O'Donnell and X. Gao, Nat. Commun., 2010, 1(4), 41.

198 L. Josephson, M. F. Kircher, U. Mahmood, Y. Tang and R. Weissleder, Bioconjugate Chem., 2002, 13(3), 554-560.

199 B. R. Jarrett, B. Gustafsson, D. L. Kukis and A. Y. Louie, Bioconjugate Chem., 2008, 19(7), 1496-1504.

200 C. M. Lee, H. J. Jeong, E. M. Kim, D. W. Kim, S. T. Lim, H. T. Kim, I. K. Park, Y. Y. Jeong, J. W. Kim and M. H. Sohn, Magn. Reson. Med., 2009, 62(6), 1440-1446.

201 J. E. Smith, C. D. Medley, Z. W. Tang, D. Shangguan, C. Lofton and W. H. Tan, Anal. Chem., 2007, 79(8), 3075-3082.

202 P. Pradhan, J. Giri, F. Rieken, C. Koch, O. Mykhaylyk, M. Doblinger, R. Banerjee, D. Bahadur and C. Plank, J. Controlled Release, 2010, 142(1), 108-121.

203 E. R. Cintra, F. S. Ferreira, J. L. Santos, J. C. Campello, L. M. Socolovsky, E. M. Lima and A. F. Bakuzis, Nanotechnology, 2009, 20(4), 045103.

204 Y. J. Chen, A. Bose and G. D. Bothun, ACS Nano, 2010, 4(6), 32153221.

205 S. Nappini, F. B. Bombelli, M. Bonini, B. Norden and P. Baglioni, Soft Matter, 2010, 6(1), 154-162. 
206 E. Amstad, S. Zurcher, A. Mashaghi, J. Y. Wong, M. Textor and E. Reimhult, Small, 2009, 5(11), 1334-1342.

207 E. Amstad, T. Gillich, I. Bilecka, M. Textor and E. Reimhult, Nano Lett., 2009, 9(12), 4042-4048.

208 J. Yang, J. Gunn, S. R. Dave, M. Q. Zhang, Y. A. Wang and X. H. Gao, Analyst, 2008, 133(2), 154-160.

209 S. Santra, C. Kaittanis, J. Grimm and J. M. Perez, Small, 2009, 5 (16), 1862-1868.

210 Z. P. Xiao, K. M. Yang, H. Liang and J. Lu, J. Polym. Sci., Part A. Polym. Chem., 2010, 48(3), 542-550.

211 N. Kohler, G. E. Fryxell and M. Q. Zhang, J. Am. Chem. Soc., 2004, 126(23), 7206-7211.

212 E. K. U. Larsen, T. Nielsen, T. Wittenborn, H. Birkedal, T. VorupJensen, M. H. Jakobsen, L. Ostergaard, M. R. Horsman, F. Besenbacher, K. A. Howard and J. Kjems, ACS Nano, 2009, 3 (7), 1947-1951.

213 M. Kim, Y. F. Chen, Y. C. Liu and X. G. Peng, Adv. Mater., 2005, 17(11), 1429.

214 M. A. White, J. A. Johnson, J. T. Koberstein and N. J. Turro, J. Am. Chem. Soc., 2006, 128(35), 11356-11357.

215 C. J. Xu, K. M. Xu, H. W. Gu, R. K. Zheng, H. Liu, X. X. Zhang, Z. H. Guo and B. Xu, J. Am. Chem. Soc., 2004, 126(32), 9938-9939.

216 E. Y. Sun, L. Josephson and R. Weissleder, Mol. Imaging, 2006, 5(2), $122-128$.

217 A. Natarajan, C. Y. Xiong, C. Gruettner, G. L. DeNardo and S. J. DeNardo, Cancer Biother. Radiopharm., 2008, 23(1), 82-91.

218 Y. W. Jun, Y. M. Huh, J. S. Choi, J. H. Lee, H. T. Song, S. Kim, S. Yoon, K. S. Kim, J. S. Shin, J. S. Suh and J. Cheon, J. Am. Chem. Soc., 2005, 127(16), 5732-5733.

219 O. Veiseh, J. W. Gunn, F. M. Kievit, C. Sun, C. Fang, J. S. H. Lee and M. Q. Zhang, Small, 2009, 5(2), 256-264.

220 O. Veiseh, F. M. Kievit, C. Fang, N. Mu, S. Jana, M. C. Leung, H. Mok, R. G. Ellenbogen, J. O. Park and M. Zhang, Biomaterials, 2010, 31(31), 8032-8042.

221 K. Chen, J. Xie, H. Y. Xu, D. Behera, M. H. Michalski, S. Biswal, A. Wang and X. Y. Chen, Biomaterials, 2009, 30(36), 6912-6919.

222 J. Xie, K. Chen, J. Huang, S. Lee, J. H. Wang, J. Gao, X. G. Li and X. Y. Chen, Biomaterials, 2010, 31(11), 3016-3022.

223 H. Y. Lee, Z. Li, K. Chen, A. R. Hsu, C. J. Xu, J. Xie, S. H. Sun and X. Y. Chen, J. Nucl. Med., 2008, 49(8), 1371-1379.

224 H. W. Gu, Z. M. Yang, J. H. Gao, C. K. Chang and B. Xu, J. Am. Chem. Soc., 2005, 127(1), 34-35.

225 H. W. Gu, K. M. Xu, Z. M. Yang, C. K. Chang and B. Xu, Chem. Commun., 2005, (34), 4270-4272.

226 R. Hong, N. O. Fischer, T. Emrick and V. M. Rotello, Chem. Mater., 2005, 17(18), 4617-4621.

227 C. Duanmu, I. Saha, Y. Zheng, B. M. Goodson and Y. Gao, Chem. Mater., 2006, 18(25), 5973-5981.

228 J. Xie, C. J. Xu, Z. C. Xu, Y. L. Hou, K. L. Young, S. X. Wang, N. Pourmond and S. H. Sun, Chem. Mater, 2006, 18(23), 54015403.

229 J. Xie, C. Xu, N. Kohler, Y. Hou and S. Sun, Adv. Mater., 2007, 19 (20), 3163.

230 J. H. Gao, G. L. Liang, J. S. Cheung, Y. Pan, Y. Kuang, F. Zhao, B. Zhang, X. X. Zhang, E. X. Wu and B. Xu, J. Am. Chem. Soc., 2008, 130(35), 11828-11833.

231 K. Somaskandan, T. Veres, M. Niewczas and B. Simard, New J. Chem., 2008, 32(2), 201-209.

232 K. H. Bae, Y. B. Kim, Y. Lee, J. Hwang, H. Park and T. G. Park, Bioconjugate Chem., 2010, 21(3), 505-512.

233 H. Basti, L. Ben Tahar, L. S. Smiri, F. Herbst, M. J. Vaulay, F. Chau, S. Ammar and S. Benderbous, J. Colloid Interface Sci., 2010, 341(2), 248-254.

234 A. S. Goldmann, C. Schödel, A. Walther, J. Yuan, K. Loos and A. H. E. Müller, Macromol. Rapid Commun., 2010.

235 L. Isa, E. Amstad, M. Textor and E. Reimhult, Chimia, 2010, 64(3), $145-149$.

236 N. Kemikli, H. Kavas, S. Kazan, A. Baykal and R. Ozturk, J. Alloys Compd., 2010, 502(2), 439-444.

237 L. Zhang, H. Xue, C. L. Gao, L. Carr, J. N. Wang, B. C. Chu and S. Y. Jiang, Biomaterials, 2010, 31(25), 6582-6588.

238 H. Bin Na, I. S. Lee, H. Seo, Y. I. Park, J. H. Lee, S. W. Kim and T. Hyeon, Chem. Commun., 2007, (48), 5167-5169.

239 T. Georgelin, B. Moreau, N. Bar, D. Villemin, V. Cabuil and O. Horner, Sens. Actuators, B, 2008, 134(2), 451-454.
240 A. Hofmann, S. Thierbach, A. Semisch, A. Hartwig, M. Taupitz, E. Ruhl and C. Graf, J. Mater. Chem., 2010, 20(36), 7842-7853.

241 J. Zhang, S. Rana, R. S. Srivastava and R. D. K. Misra, Acta Biomater., 2008, 4(1), 40-48.

242 R. Narain, M. Gonzales, A. S. Hoffman, P. S. Stayton and K. M. Krishnan, Langmuir, 2007, 23(11), 6299-6304.

243 M. D. Butterworth, L. Illum and S. S. Davis, Colloids Surf., A, 2001, 179(1), 93-102.

244 Y. Zhang, N. Kohler and M. Q. Zhang, Biomaterials, 2002, 23(7), $1553-1561$

245 O. Veiseh, C. Sun, J. Gunn, N. Kohler, P. Gabikian, D. Lee, N. Bhattarai, R. Ellenbogen, R. Sze, A. Hallahan, J. Olson and M. Q. Zhang, Nano Lett., 2005, 5(6), 1003-1008.

246 C. Sun, R. Sze and M. Q. Zhang, J. Biomed. Mater. Res., Part A, 2006, 78(3), 550-557.

247 R. De Palma, S. Peeters, M. J. Van Bael, H. Van den Rul, K. Bonroy, W. Laureyn, J. Mullens, G. Borghs and G. Maes, Chem. Mater., 2007, 19(7), 1821-1831.

248 Y. Zhou, S. X. Wang, B. J. Ding and Z. M. Yang, Chem. Eng. J., 2008, 138(1-3), 578-585.

249 K. Herve, L. Douziech-Eyrolles, E. Munnier, S. Cohen-Jonathan, M. Souce, H. Marchais, P. Limelette, F. Warmont, M. L. Saboungi, P. Dubois and I. Chourpa, Nanotechnology, 2008, 465608, (7 pp).

250 B. Y. Du, A. X. Mei, P. J. Tao, B. Zhao, Z. Cao, J. J. Nie, J. T. Xu and Z. Q. Fan, J. Phys. Chem. C, 2009, 113(23), 10090-10096.

251 Z. P. Chen, R. Z. Xu, Y. Zhang and N. Gu, Nanoscale Res. Lett., 2009, 4(3), 204-209.

252 C. Barrera, A. P. Herrera and C. Rinaldi, J. Colloid Interface Sci., 2009, 329(1), 107-113.

253 C. R. Sun, K. Du, C. Fang, N. Bhattarai, O. Veiseh, F. Kievit, Z. Stephen, D. H. Lee, R. G. Ellenbogen, B. Ratner and M. Q. Zhang, ACS Nano, 2010, 4(4), 2402-2410.

254 D. Forge, S. Laurent, Y. Gossuin, A. Roch, L. V. Elst and R. N. Muller, J. Magn. Magn. Mater., 2011, 323(5), 410-415.

255 Y. X. J. Wang, S. M. Hussain and G. P. Krestin, Eur. Radiol., 2001, 11(11), 2319-2331.

256 C. W. Jung and P. Jacobs, Magn. Reson. Imaging, 1995, 13(5), 661674.

257 R. Weissleder, G. Elizondo, J. Wittenberg, C. A. Rabito, H. H. Bengele and L. Josephson, Radiology, 1990, 175(2), 489-493.

258 E. Amstad; L. W. E. Starmans, M. A. Visbal, L. Isa, C. Rinaldi, M. Textor and E. Reimhult, in preparation.

259 A. Tsourkas, V. R. Shinde-Patil, K. A. Kelly, P. Patel, A. Wolley, J. R. Allport and R. Weissleder, Bioconjugate Chem., 2005, 16(3), $576-581$.

260 K. A. Kelly, J. R. Allport, A. Tsourkas, V. R. Shinde-Patil, L. Josephson and R. Weissleder, Circ. Res., 2005, 96(3), 327-336.

261 M. Nahrendorf, F. A. Jaffer, K. A. Kelly, D. E. Sosnovik, E. Aikawa, P. Libby and R. Weissleder, Circulation, 2006, 114(14), 1504-1511.

262 A. L. Martin, L. M. Bernas, B. K. Rutt, P. J. Foster and E. R. Gillies, Bioconjugate Chem., 2008, 19(12), 2375-2384.

263 A. L. Martin, J. L. Hickey, A. L. Ablack, J. D. Lewis, L. G. Luyt and E. R. Gillies, J. Nanopart. Res., 2010, 12(5), 1599-1608.

264 Y. M. Huh, Y. W. Jun, H. T. Song, S. Kim, J. S. Choi, J. H. Lee, S. Yoon, K. S. Kim, J. S. Shin, J. S. Suh and J. Cheon, J. Am. Chem. Soc., 2005, 127(35), 12387-12391.

265 J. Meng, J. Fan, G. Galiana, R. T. Branca, P. L. Clasen, S. Ma, J. Zhou, C. Leuschner, C. Kumar, J. Hormes, T. Otiti, A. C. Beye, M. P. Harmer, C. J. Kiely, W. Warren, M. P. Haataja and W. O. Soboyejo, Mater. Sci. Eng., C, 2009, 29(4), 1467-1479.

266 J. I. Cutler, D. Zheng, X. Y. Xu, D. A. Giljohann and C. A. Mirkin, Nano Lett., 2010, 10(4), 1477-1480.

267 M. Funovics, X. Montet, F. Reynolds, R. Weissleder and L. Josephson, Neoplasia, 2005, 7(10), 904-911.

268 R. Weissleder, K. Kelly, E. Y. Sun, T. Shtatland and L. Josephson, Nat. Biotechnol., 2005, 23(11), 1418-1423.

269 X. Montet, M. Funovics, K. Montet-Abou, R. Weissleder and L. Josephson, J. Med. Chem., 2006, 49(20), 6087-6093.

270 D. R. Elias, Z. L. Cheng and A. Tsourkas, Small, 2010, 6(21), 24602468.

271 G. R. Reddy, M. S. Bhojani, P. McConville, J. Moody, B. A. Moffat, D. E. Hall, G. Kim, Y. E. L. Koo, M. J. Woolliscroft, J. V. Sugai, T. D. Johnson, M. A. Philbert, 
R. Kopelman, A. Rehemtulla and B. D. Ross, Clin. Cancer Res., 2006, 12(22), 6677-6686.

272 D. Simberg, T. Duza, J. H. Park, M. Essler, J. Pilch, L. L. Zhang A. M. Derfus, M. Yang, R. M. Hoffman, S. Bhatia, M. J. Sailor and E. Ruoslahti, Proc. Natl. Acad. Sci. U. S. A., 2007, 104(3), 932-936.

273 F. Sonvico, S. Mornet, S. Vasseur, C. Dubernet, D. Jaillard, J. Degrouard, J. Hoebeke, E. Duguet, P. Colombo and P. Couvreur, Bioconjugate Chem., 2005, 16(5), 1181-1188.

274 C. F. Zhang, M. Jugold, E. C. Woenne, T. Lammers, B. Morgenstern, M. M. Mueller, H. Zentgraf, M. Bock, M. Eisenhut, W. Semmler and F. Kiessling, Cancer Res., 2007, 67 (4), 1555-1562.

275 X. B. Li, X. K. Du, T. L. Huo, X. Liu, S. Zhang and F. Yuan, Acta Radiol., 2009, 50(6), 583-594.

276 J. K. Zhou, C. Leuschner, C. Kumar, J. F. Hormes and W. O. Soboyejo, Biomaterials, 2006, 27(9), 2001-2008.

277 P. R. Reynolds, D. J. Larkman, D. O. Haskard, J. V. Hajnal, N. L. Kennea, A. J. T. George and A. D. Edwards, Radiology, 2006, 241(2), 469-476.

278 S. Boutry, S. Laurent, L. Vander Elst and R. N. Muller, Contrast Media Mol. Imaging, 2006, 1(1), 15-22.
279 C. Burtea, S. Laurent, A. Roch, L. Vander Elst and R. N. Muller, J. Inorg. Biochem., 2005, 99(5), 1135-1144.

280 M. K. Yu, J. Park, Y. Y. Jeong, W. K. Moon and S. Jon, Nanotechnology, 2010, 21(41), 415102.

281 M. V. Yigit, D. Mazumdar, H. K. Kim, J. H. Lee, B. Dintsov and Y. Lu, ChemBioChem, 2007, 8, 1675-1678.

282 A. M. Koch, F. Reynolds, H. R. Merkle, R. Weissleder and L. Josephson, ChemBioChem, 2005, 6(2), 337-345.

283 L. Wang, K. G. Neoh, E. T. Kang, B. Shuter and S. C. Wang, Biomaterials, 2010, 31(13), 3502-3511.

284 M. J. Pittet, F. K. Swirski, F. Reynolds, L. Josephson and R. Weissleder, Nat. Protoc., 2006, 1, 73-79.

285 Z. Medarova, N. V. Evgenov, G. Dai, S. Bonner-Weir and A. Moore, Nat. Protoc., 2006, 1(1), 429-435.

286 M. Nahrendorf, H. W. Zhang, S. Hembrador, P. Panizzi, D. E. Sosnovik, E. Aikawa, P. Libby, F. K. Swirski and R. Weissleder, Circulation, 2008, 117(3), 379-387.

287 B. R. Jarrett, C. Correa, K. L. Ma and A. Y. Louie, PLoS One, 2010, 5(10), e13254.

288 B. R. Jarrett, B. Gustafsson, D. L. Kukis and A. Y. Louie, Bioconjugate Chem., 2008, 19(7), 1496-1504. 